\title{
EzGET: A LIBRARY OF FORTRAN SUBROUTINES TO FACILITATE DATA RETRIEVAL
}

by

Karl E. Taylor

Program for Climate Model Diagnosis and Intercomparison Lawrence Livermore National Laboratory, Livermore, CA, USA 


\section{DISCLAIMER}

This document was prepared as an account of work sponsored by an agency of the United States Government. Neither the United States Government nor the University of California nor any of their employees, makes any warranty, express or implied, or assumes any legal liability or responsibility for the accuracy, completeness, or usefulness of any information, apparatus, product, or process disclosed, or represents that its use would not infringe privately owned rights. Reference herein to any specific commercial product, process, or service by trade name, trademark, manufacturer, or otherwise, does not necessarily constitute or imply its endorsement, recommendation, or favoring by the United States Government or the University of California. The views and opinions of authors expressed herein do not necessarily state or reflect those of the United States Government or the University of California, and shall not be used for advertising or product endorsement purposes.

This is an informal report intended primarily for internal or limited external distribution. The opinions and conclusions stated are those of the author and may or may not be those of the Laboratory.

This report has been reproduced directly from the best available copy.

Available to DOE and DOE contractors from the Office of Scientific and Technical Information P.O. Box 62, Oak Ridge, TN 37831

Prices available from (615) 576-8401, FTS 626-8401

Available to the public from the National Technical Information Service

U.S. Department of Commerce 5285 Port Royal Rd. Springfield, VA 22161 


\section{EzGet: \\ A Library of Fortran Subroutines to Facilitate Data Retrieval}

by

Karl E. Taylor

Program for Climate Model Diagnosis and Intercomparison
Lawrence Livermore National Laboratory, Livermore. CA. USA

DISCLAIMER

This report was prepared as an account of work sponsored by an agency of the United States Government. Neither the United States Government nor any agency thereof, nor any of their employees, makes any warranty, express or implied, or assumes any legal liability or responsibility for the accuracy, completeness, or usefulness of any information, apparatus, product, or process disclosed, or represents that its use would not infringe privately owned rights. Reference herein to any specific commercial product, process, or service by trade name, trademark, manufacturer, or otherwise does not necessarily constitute or imply its endorsement, recommendation, or favoring by the United States Government or any agency thereof. The views and opinions of authors expressed herein do not necessarily state or reflect those of the United States Government or any agency thereof. 


\section{DISCLAIMER}

Portions of this document may be illegible in electronic image products. Images are produced from the best available original document. 


\section{Contents}

Abstract $\quad$ v

1 Introduction $\quad 1$

2 Tutorial Examples $\quad 2$

2.1 Simple retrieval of data . . . . . . . . . . . . . 2

2.2 Masking geographical regions when retrieving data . . . . . . 10

2.3 Mapping data to a specified grid . . . . . . . . . . . 13

2.4 Computing an area average ................... 17

2.5 A typical amip application ....................... 19

2.6 Placing data in contiguous memory .............. 25

2.7 Reducing memory requirements . . . . . . . . . . . . . 28

2.8 Another example of area-averaging ................. 32

$\begin{array}{lll}3 & \text { EzGet Subroutines } & \mathbf{3 6}\end{array}$

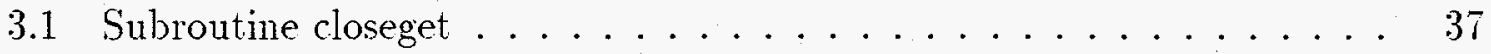

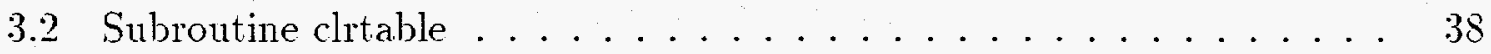

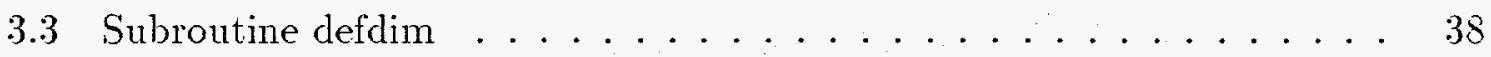

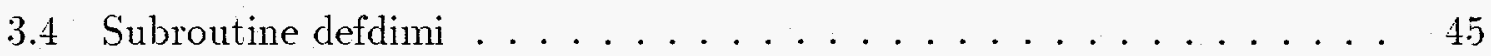

3.5 Subroutine defgeog .......................... 46

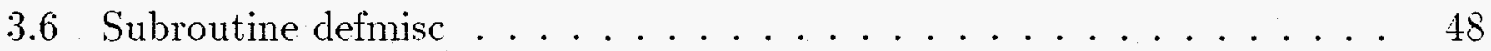

3.7 Subroutine defregrd . . . . . . . . . . . . . 50

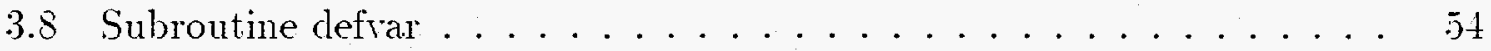

3.9 Subroutine defvarex . . . . . . . . . . . . . . . . 55

3.10 Subroutine domain . . . . . . . . . . . . . . . 56

3.11 Subroutine domlimit . . . . . . . . . . . . . . 56 
3.12 Subroutine getcoord $\ldots \ldots \ldots \ldots \ldots \ldots \ldots \ldots \ldots$

3.13 Subroutine getdata . . . . . . . . . . . . 5 i

3.14 Subroutine getdimwt . . . . . . . . . . . . . 58

3.15 Subroutine getedges . . . . . . . . . . . . . . . . 59

3.16 Subroutine getfield $\ldots \ldots \ldots \ldots \ldots \ldots$

3.17 Subroutine getgeog . . . . . . . . . . . . . . 60 60

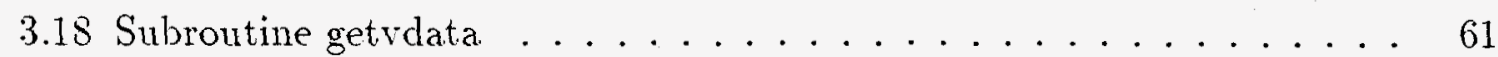

3.19 Subroutine initget . . . . . . . . . . . . . 62

3.20 Subroutine lendims . . . . . . . . . . . . . . 62

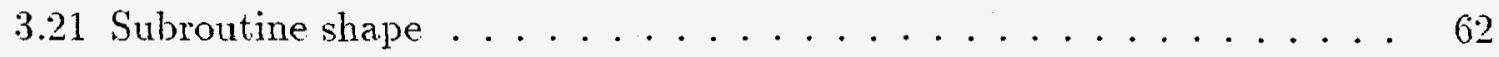

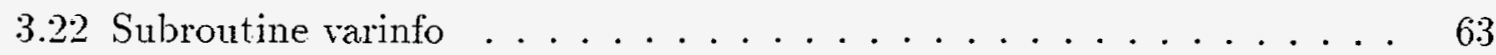

4 Avoiding Errors $\quad 64$

4.1 Input/output devices . . . . . . . . . . . . . . . . 64

4.2 Subroutine and common names . . . . . . . . . . . 64

$4.3 \quad$ EzGet size limits . . . . . . . . . . . . . . . 6.5

5 Obtaining and Installing EzGet Software $\quad 65$

$\begin{array}{lll}\text { Appendix A Model Acronyms and Associated Weights } & 67\end{array}$

$\begin{array}{lll}\text { Appendix B Geographical Regions } & \mathbf{7 0}\end{array}$ 


\begin{abstract}
The software described in this document is designed to facilitate retrieval of modeled and observed climate data stored in popular formats including DRS, netCDF, GrADS, and, if a control file is supplied, GRIB. You can specify how the data should be structured and whether it should undergo a grid transformation before you receive it, even when you know little about the structure of the stored data (i.e., its dimension order, grid, and domain).

The software is referred to here as EzGet (pronounced "easy-get") and it comprises a set of subroutines that can be linked to any FORTRAN program. EzGet reads files through the colunif interface which is available from the Program for Climate Model Diagnosis and Intercomparison (PCMDI), but use of EzGet does not require familiarity with cdunif. The main advantages of using this software instead of the lower level cdunif library include:
\end{abstract}

- Substantial error trapping capabilities and detailed error messages

- Versatile capability of conveniently selecting data from specified regions (e.g., oceans, North America, all land areas north of 45 degrees latitude, etc.)

- Ability to map data to a new grid at the time it is retrieved by EzGet

- Automatic creation of "weights" for use in subsequent averaging or masking of data.

- Increased control in specifying the domain, grid and structure of the retrieved data.

Taken together these capabilities will simplify the process of writing programs for accessing data stored in different formats and structures, including all the observed data. sets and the model output from various model intercomparison projects (AMIP, PMIP. CMIP, etc.) archived at PCMDI.

EzCret software and the latest version of this document are arailable through the PCMDI web site:

home page: http://www-pcmdi.llnl.gov/

EzGet location: ht.tp://www-pcmdi.lnl.gov/ktaylor/ezget/ezget.html 


\section{Introduction}

It is usually not too difficult to write a FORTRAN program that performs the same series of calculations on several different sets of data, as long as all the data are stored on the same grid and in the same structure and format. If, however, data are found in different formats and structures, then the program can become quite complex, or will have to be revised to treat each new set of data. The software described here makes it easier to write programs that can accept data sets stored in a variety of structures. It can retrieve data that have been stored in the following cdunif-accessible formats: DRS, netCDF, GrADS, and GRIB (if a control file is supplied). It is designed to be especially useful in analyzing output from climate models. These models may have different grids, the data may be stored in different orders (e.g., south to north vs. north to south), and the dimensions may have slightly different names (e.g., "longitude" vs. "Longitude").

A powerful feature of this software is that it can both retrieve data from precisely specified domains and also map data to a common grid (specified by the user or taken from another file) so that point-wise intercomparisons between model results and between modeled and observed fields can be carried out without difficulty. Furthermore, you can automatically obtain the "weight" (which commonly is the grid-cell area) associated with each grid cell. The set of weights associated with an array of data make it easy to compute mean values (or other area-weighted statistics) and avoid the use of "if" tests in cases when data might be "missing" from some grid cells. Finally. for the models included in the Atmospheric Model Intercomparison Project (AMIP) and Paleoclimate Modeling Intercomparison Project (PMIP), special gridded maps are arailable from PCMDI (Program for Climate Model Diagnosis and Intercomparison) that allow one to retrieve data from individual geographical regions (e.g.. North America. Indian Ocean, etc.). All the data archived at PCMDI, including the AMIP and PMIP model standard output, is accessible through this software. EzGet is limited to retrieving (multi-dimensional) rectangular arrays of data, but there is complete control over the limits of the domain, and with EzGet's masking capabilities, non-rectangular subsets of the domain can be selected.

EzGet serves a different purpose from other software tools developed at PCMDI. It is meant to be used in conjunction with FORTRAN programs that need access to data. For direct viewing and interactive manipulation of data. a Visualization and Computation System (VCS) has been developed. To transfer data to files. formats, or visualization systems, the Data and Dimensions Interface (DDI) has been developed. Neither of these can be called from a FORTRAN program.

As with any new software, some effort will be required to become familiar with Ez iet.'s capabilities and to learn how to apply it. In the next section several tutorial examples are given that begin this process. 


\section{Tutorial Examples}

With the help of $\mathrm{EzGet}$, data can be retrieved from files (in formats readable by cdunif) through a set of subroutine calls that are described in this document. Parameters that control such capabilities as specifying precise domains, mapping data to specified grids, and masking various regions are set through subroutine calls. Typical applications of this software are illustrated by the following examples. The first example should be studied with some thoroughness, because it provides the basis for the subsequent examples.

\subsection{Simple retrieval of data}

Suppose we want to retrieve the surface air temperature data contained in a single file. The following short program accomplishes this: ${ }^{1}$

\section{program extract}

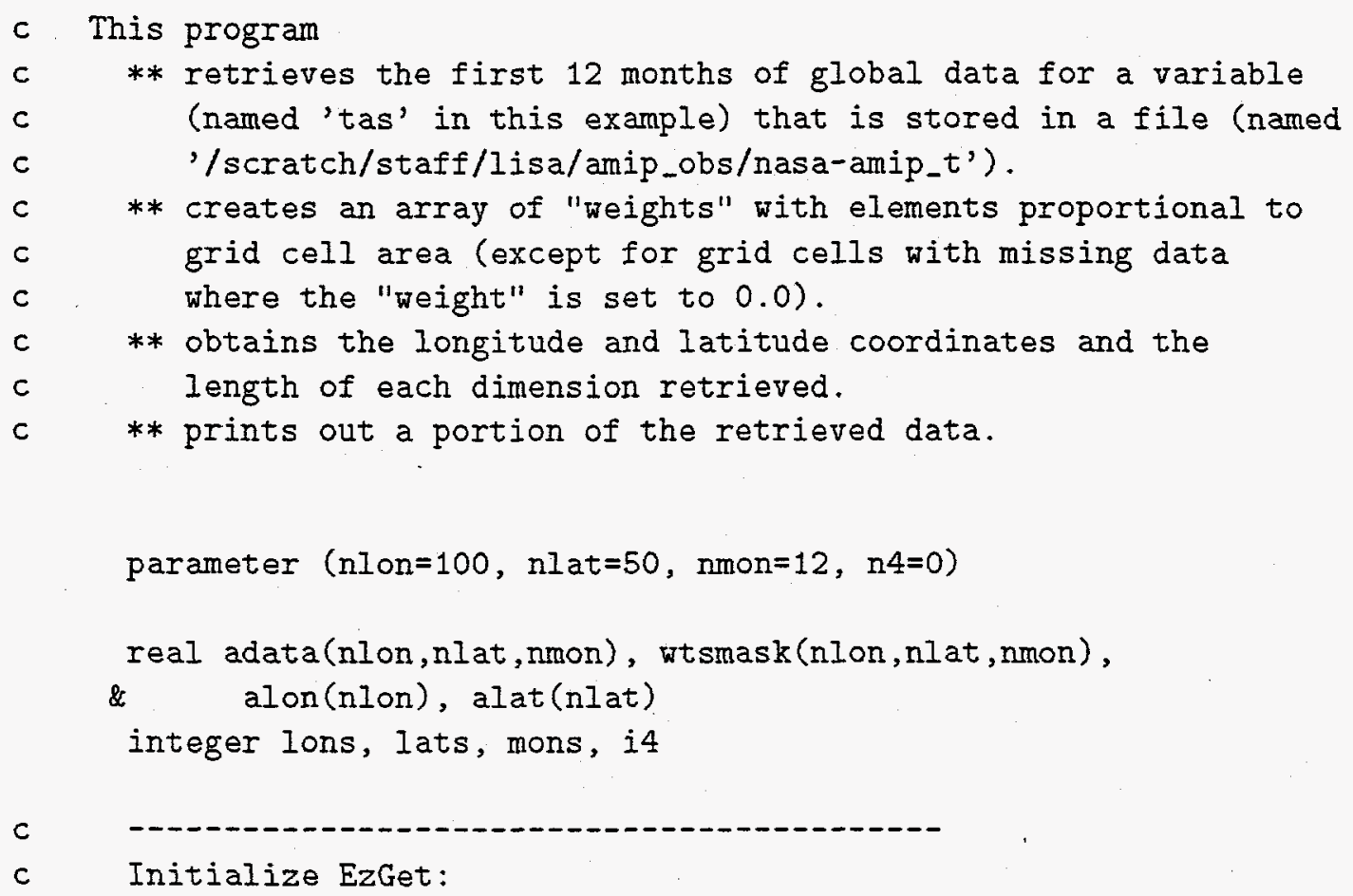

\footnotetext{
${ }^{1}$ Further information about each of the Ezcret subroutines can be found later in this section where a more completely annotated version of program extract appears.
} 


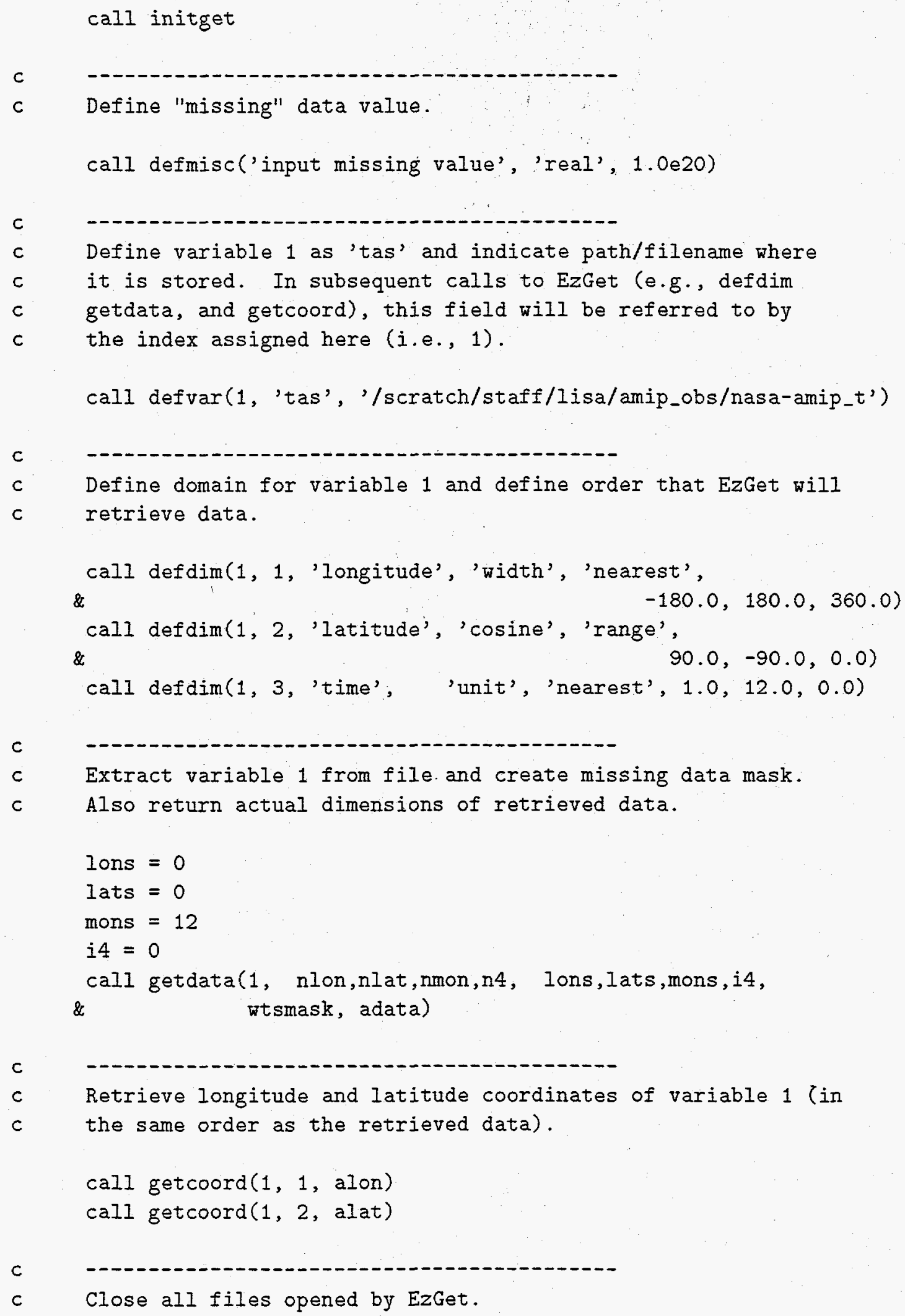




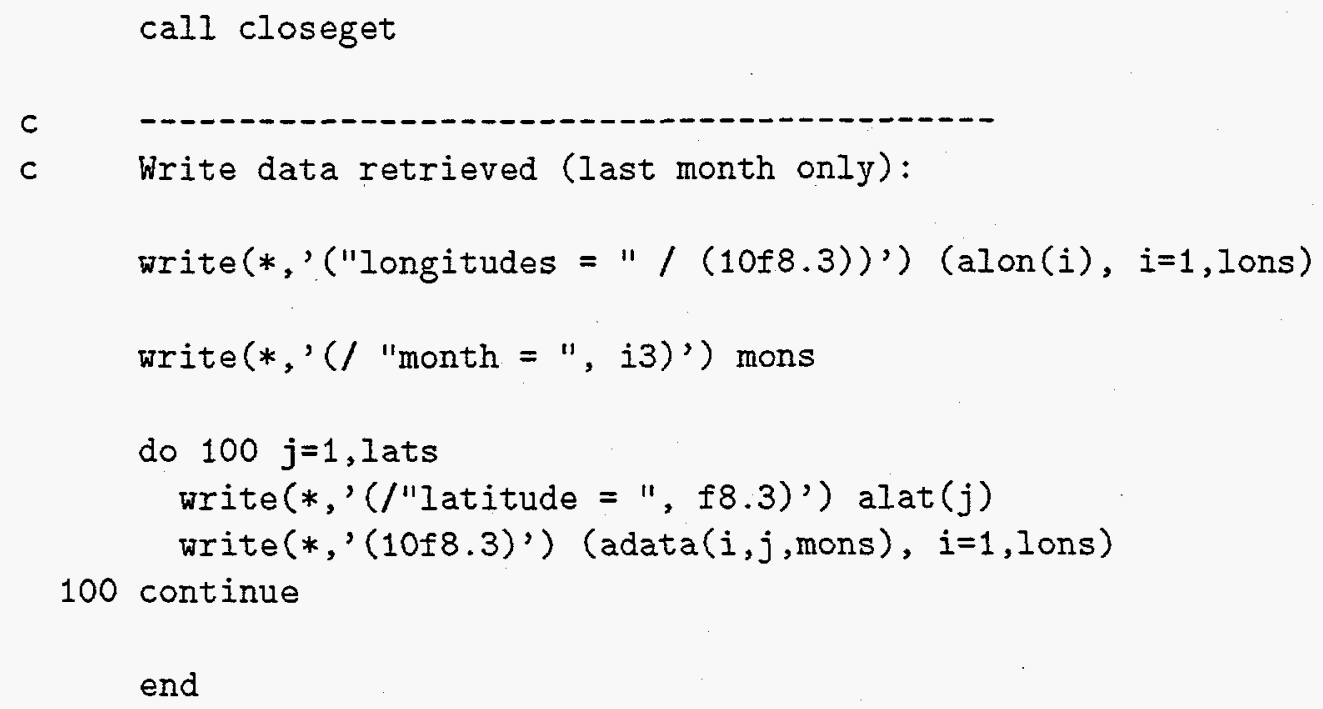

A fully annotated copy of this program appears below and each of the seven subroutines called by it is documented in Section 3, but here we briefly describe the purpose of each of these subroutines:

initget .... This subroutine must be called to initialize EzGet. It assigns default values to a few parameters and sets up some internal tables.

defmisc ... This subroutine ('define miscellaneous') can be used to override certain default parameters assumed by EzGet. (Note in the program above the default parameter for missing data would be set to $1.0 \mathrm{e} 20$, but it happens that this is the default, so this call to defmisc is not really necessary.)

defvar .... This subroutine defines a variable that will be referenced subsequently by a simple integer index.

defiim .... This subroutine specifies the dimension ordering along with the desired domain for the data that will be retrieved. It also provides information for creating an array of "weights," typically set proportional to the grid-cell areas, but set to 0.0 in regions of missing data.

getdata ... This subroutine retrieves data and creates an appropriate mask (or set of "weights") associated with the data.

getcoord .... This subroutine retrieves the coordinates associated with a retrieved field.

closeget .... This subroutine closes any files opened by EzGet.

Here is the fully annotated version of the above program: 
program extract

$c$

c

c

This program

** retrieves the first 12 months of global data for a variable (named 'tas' in this example) that is stored in a file (named '/scratch/staff/lisa/amip_obs/nasa-amip_t').

** creates an array of "weights" with elements proportional to grid cell area (except for grid cells with missing data where the "weight" is set to 0.0 ).

** obtains the longitude and latitude coordinates and the length of each dimension retrieved.

** prints out a portion of the retrieved data.

Concerning the structure of the stored data, we assume the following:

1. The field is a function of longitude, latitude, and time, but the dimension order is unknown.

2. The data are stored in a rectangular array.

3. The size of the array retrieved is limited as follows:

a. The longitude dimension is no longer than 100 elements.

b. The latitude dimension is no longer than 50 elements.

c. The time dimension is no longer than 12 elements.

4. The names of the dimensions (as stored in the file) are:

'latitude', 'longitude', and 'time' (but not necessarily in that order).

5. The longitude coordinates are evenly spaced.

6. The latitude coordinates are evenly spaced.

7. The units for longitude and latitude are degrees.

8. Only one field in the file has the name, 'tas' (i.e., this variable name is unique in the file).

9. The number, $1.0 e 20$, is stored in the place of the true data anywhere that data are missing (i.e., this is the "missing data" value or indicator).

This program obtains the following information:

lons $=$ the actual length of the longitude dimension of the data retrieved from storage.

lats = the actual length of the latitude dimension of the data retrieved from storage.

mons $=$ the actual number of months of data retrieved from storage. alon $(100)=$ a vector containing the longitude coordinates for the data. 


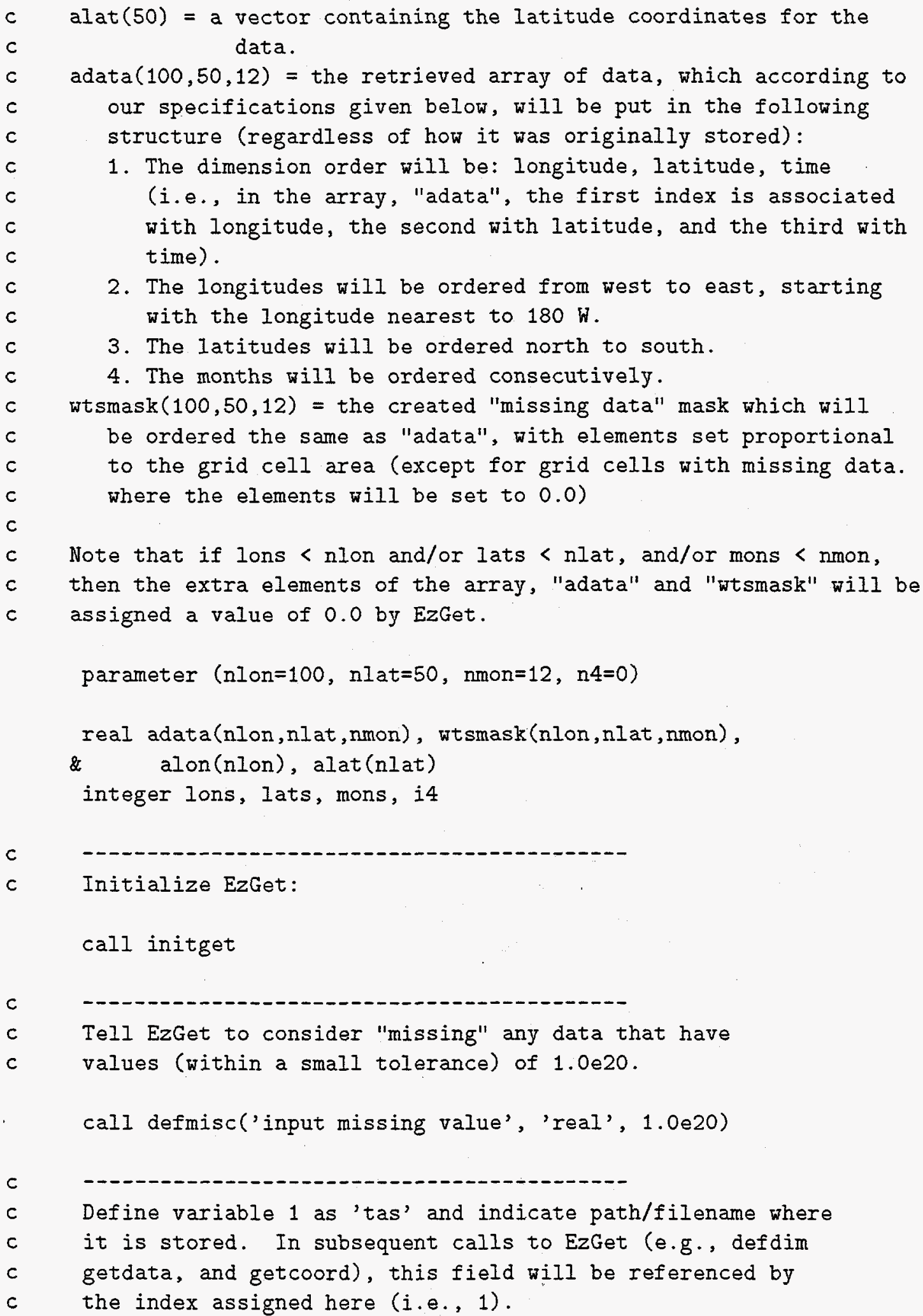


call defvar(1, 'tas', '/scratch/staff/lisa/amip_obs/nasa-amip_t')

c

c

c

Define domain for variable 1 and define order that EzGet will retrieve data.

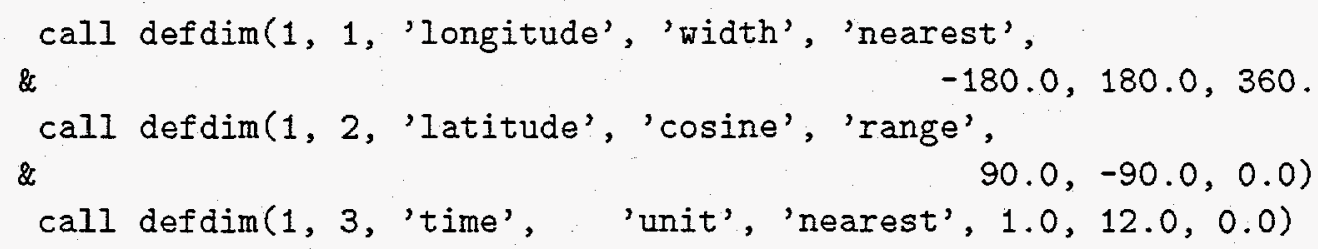


'cosine': this option for controlling creation of weights specifies that weights be generated equal approximately to the cosine of latitude (i.e., abs( $\sin ($ bdry1) sin(bdry2)), where bdry 1 and bdry2 are edges of the latitude grid-cell, assumed to be half-way between grid-cell centers) be assigned to all elements (except the weight will be 0.0 for grid cells with missing data). Another option ('gaussian') would be appropriate for spectral models with gaussian grids (as opposed to the evenly spaced grid accessed here).

'range', $90.0,-90.0,0.0$ :

these 4 arguments define the domain that will be retrieved (and also the order of retrieval). In this case all data with latitude coordinates in the range 90 to -90 will be retrieved, starting near 90 (i.e. data will be retrieved from north to south). The value 0.0 indicates that this coordinate is not cyclical.

and in the third call to defdim above the fourth argument indicates the following:

'unit': this option for controlling creation of weights specifies that unit weight should be given to each element of this dimension (except the weight will be 0 . for grid cells with missing data).

Extract variable 1 from file and create missing data mask. The lengths of the longitude, latitude and time dimensions of the data stored in the file are unknown, so initialize the "expected" dimension lengths to 0 . Return the actual dimensions of the retrieved data.

lons $=0$

lats $=0$

mons $=12$

$i 4=0$

call getdata(1, nlon, nlat,nmon, n4, lons, lats,mons, i4, $\&$ wtsmask, adata)

where on calling getdata the arguments have been defined as follows :

1: The first argument has the value 1 and indicates that data will be extracted for variable 1 (defined in defvar above). nlon, nlat, nmon, n4: 
c

$c$

$\mathrm{C}$

c

C

C

c

c

c

c

c

c

C

$c$

C

c
These are the declared dimensions of the arrays, wtsmask and adata. Note that $n 4=0$ because these arrays have only 3 dimensions.

lons, lats, mons, i4:

In this example, the user does not know how large the actual longitude and latitude dimensions of the data being retrieved will be, so these are set to 0 . The time dimension is expected to be 12 (months) as specified in the earlier call to defdim. In general, if the user knows the size of the domain to be retrieved, it is usually prudent to set these arguments to the expected size of the domain, because then EzGet can error exit if the actual size differs from what is expected.

On return from getdata:

1, nlon, nlat, nmon, n4:

The first 5 arguments will be unchanged.

lons, lats, mons, i4:

returns the actual dimensions of the data retrieved. wtsmask: returns the weights associated with the data.

In this example, the weights will either be 0.0 or proportional to grid cell area, depending on whether or not the data are missing.

adata: returns the extracted data.

Retrieve longitude and latitude coordinates of variable 1 (in the same order as the retrieved data).

call getcoord $(1,1$, alon)

call getcoord (1, 2, alat)

where in the first call to getcoord above, the arguments indicate the following:

1: The 1st argument has the value 1 and indicates that we want to obtain the coordinates of data extracted for variable 1 (as defined in the earlier call to defvar).

1: The 2nd argument has the value 1 and indicates that we want to obtain the coordinates for the 1st dimension (as defined in the earlier call to defim).

alon: returns the coordinate values for the 1st dimension of of variable 1 (i.e., 'Iongitude', as defined by defdim). 


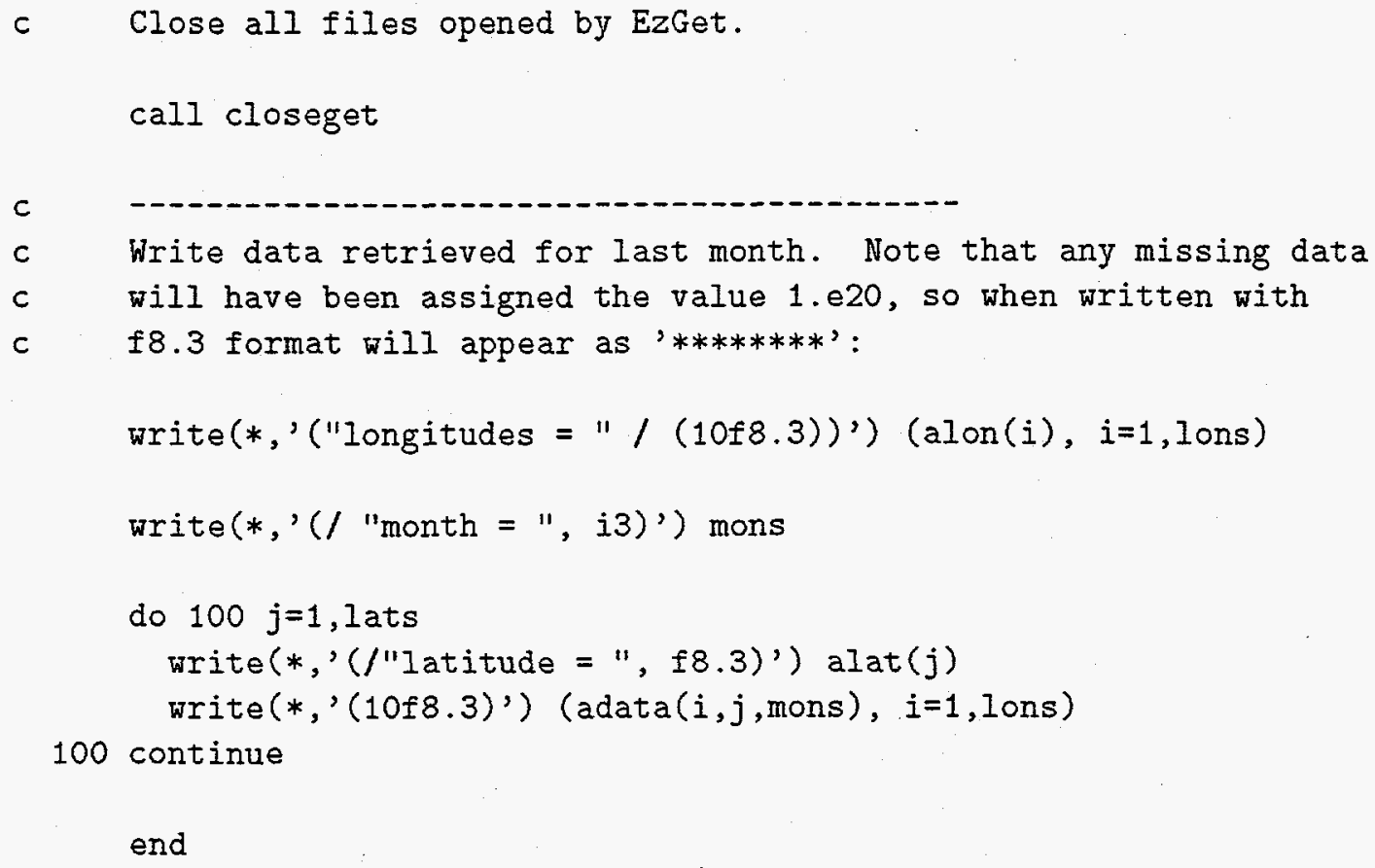

\subsection{Masking geographical regions when retrieving data}

In order to mask out data that lie outside some geographical region of interest, a file must be created containing data that uniquely identify each grid cell as belonging to a particular geographical region (such as 'land' or 'ocean' for land/sea type masks (which for AMIP data are named 'sft'), or such as 'North America', 'Indian Ocean', 'South Atlantic', etc. for region type masks (which for AMIP and PMIP data are named 'sftbyrgn'). Geography data files compatible with EzGet are available for most model grids from PCMDI. Besides the EzGet subroutines discussed in example 1, the following subroutine will also be called:

defgeog .... This subroutine makes it possible for you to specify that certain geographical regions should be masked out.

Suppose we want to obtain data from North America for the region north of the Tropic of Cancer. The following program accomplishes this task.

program getregn

c This program

c ** retrieves surface temperature data for the region of 


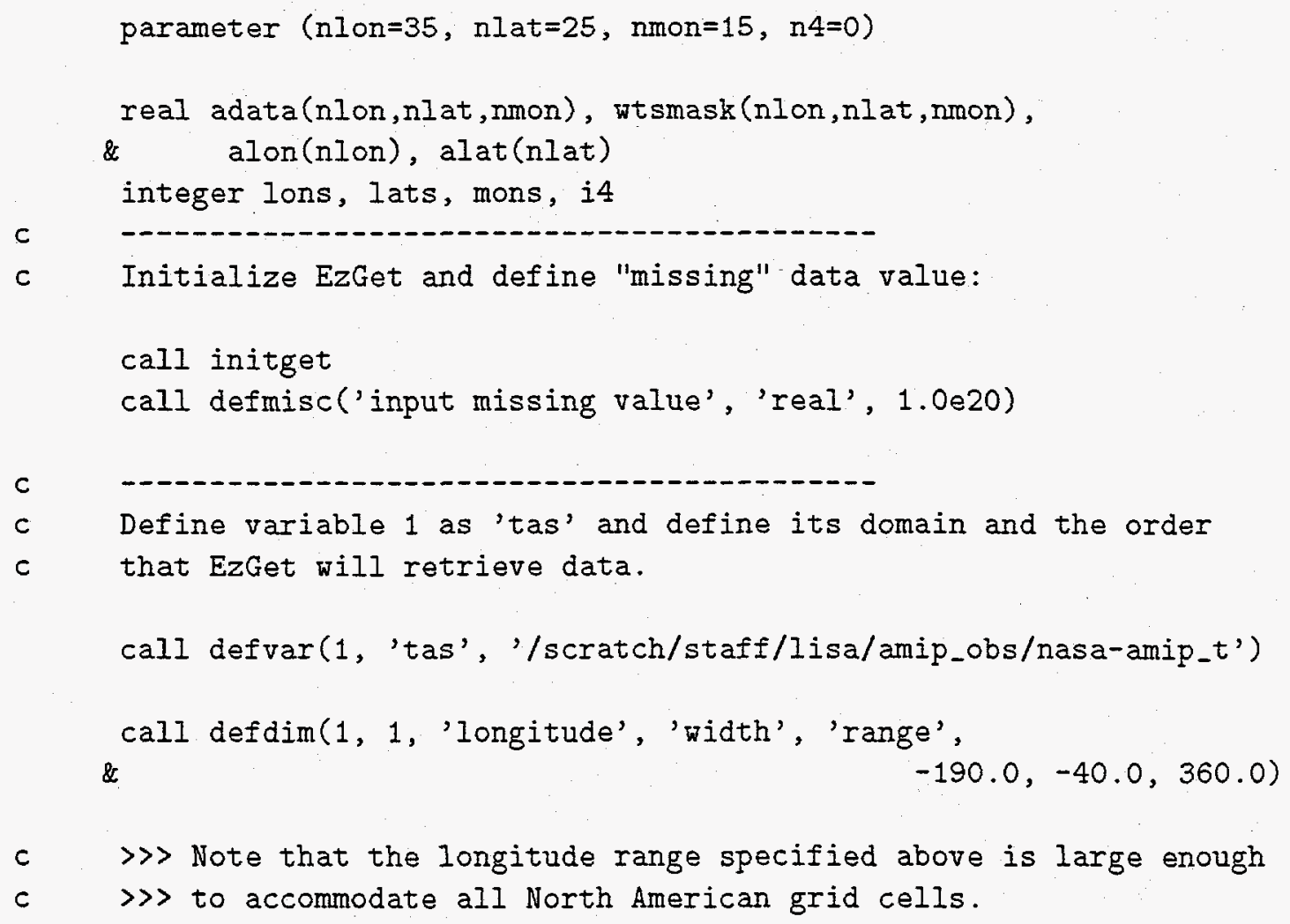
extracted only for grid cells whose coordinates are not outside the range $23.5 \mathrm{~N}$ to $90.0 \mathrm{~N}$ and $190 \mathrm{~W}$ to $40 \mathrm{~W}$.)

** creates an array of "weights", with elements set proportional to the area of the grid cells falling within the selected geographical region (except for those grid cells that contain "missing" data or which lie outside North America, in which case the weight is set to 0.0 ).

** obtains the longitude and latitude coordinates and the length of each dimension retrieved.

This program is in many ways similar to example 1 , and further explanation can be found in the comments appearing in that example (program extract).

Note that the region is selected in two ways.

First, all data for grid cells in the region $23.5 \mathrm{~N}$ to $90.0 \mathrm{~N}$ and $190 \mathrm{~W}$ and $40 \mathrm{~W}$ are extracted, and then all grid-cells that are outside the North American boundaries are masked out. The size of the arrays, adata and wtsmask, only need to be large enough to accommodate the region extracted (before the geography mask is applied).

North America north of $23.5 \mathrm{~N}$ latitude. (Note: data are 
call defdim(1, 2, 'Iatitude', 'cosine', 'range', 23.5, 90.0, 0.0)

call defdim(1, 3, 'time', 'unit', 'nearest', $1.0,12.0,0.0$ )

c $\gg$ Define variable 2 as 'sftbyrgn' and indicate path/filename

$c \quad \gg$ where it is stored. This variable should contain a "geography

c $\gg$ mask" that is compatible with the EzGet convention for

c $\gg$ identifying different geographical regions (see documentation

$c \quad \gg$ of subroutine defgeog), and it should be on the same grid as

c $\gg$ variable 1 defined above. In subsequent calls to EzGet (e.g.,

$c \quad \gg$ defgeog) this field will be referred to by the index assigned

c $\gg$ here (i.e., 2).

call defvar(2, 'sftbyrgn', '/amipsp/drs/sftbyrgn/sftbyrgn_gla') $c$

c $\gg$ Control geographical masking of retrieved data.

call defgeog(1, 'in', 2, 'North America')

c. \> where the arguments indicate the following:

C
1: The first argument has the value 1 and indicates that the geography mask will be applied to variable 1 .

'in': indicates that the masking should be done before any regridding is performed. (See later examples for further explanation.)

2: Indicates that variable 2 contains the geography data.

'North America': indicates that this is the region of interest and any data outside this region should be masked.

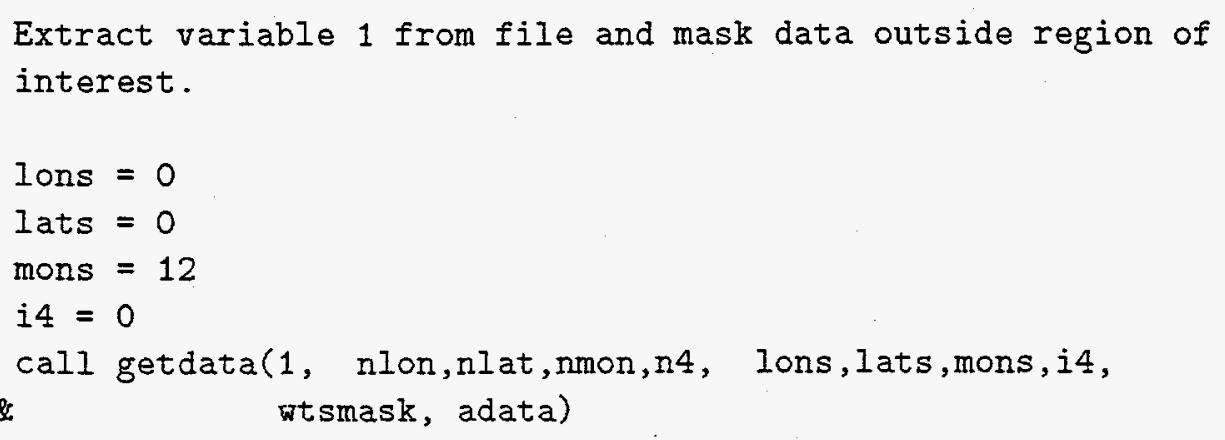




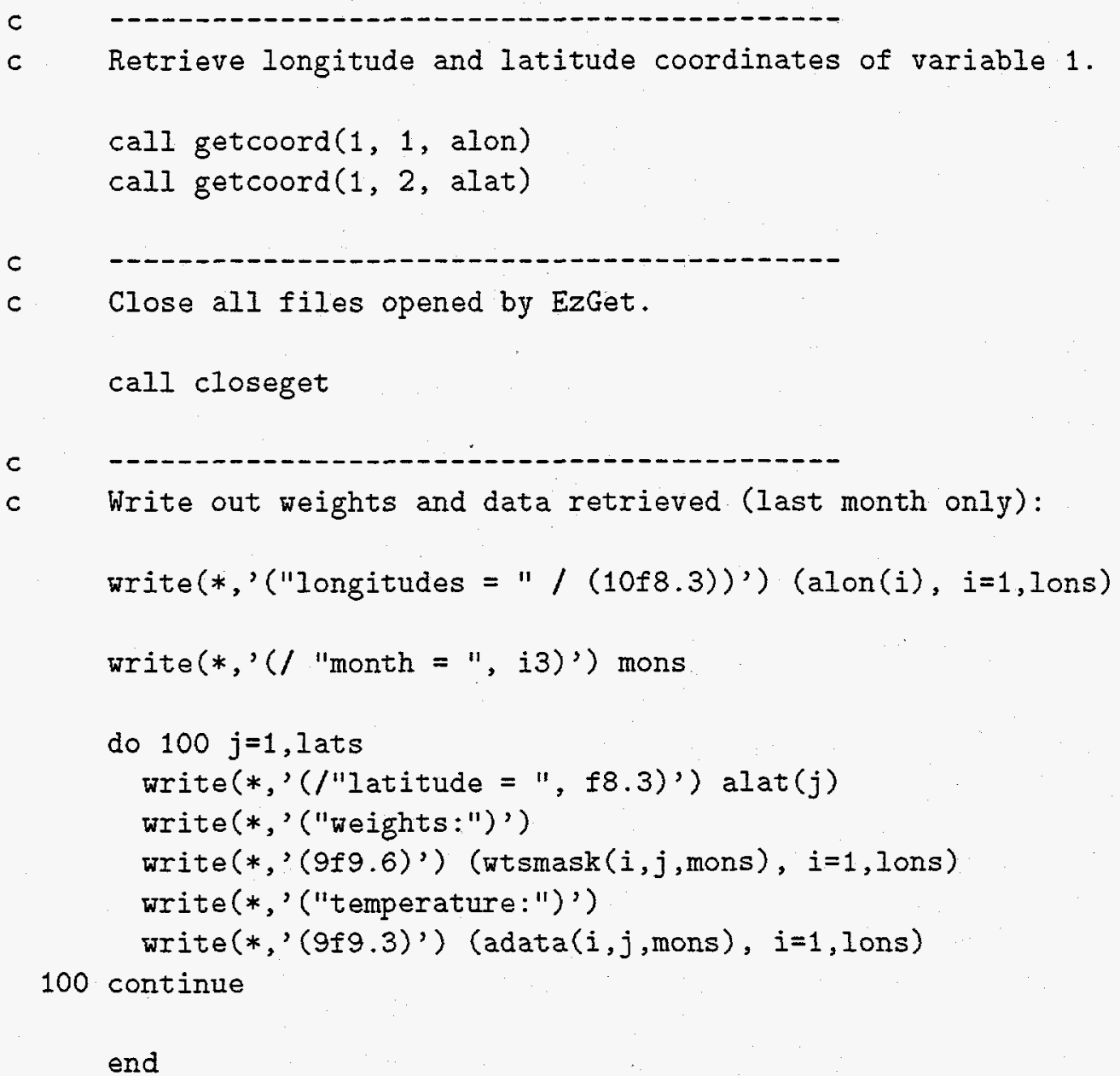

\subsection{Mapping data to a specified grid}

Suppose that data have been stored on a longitude-latitude grid that is different from the grid you might require for a particular application (e.g., to compare to data stored on a different grid). EzGet will map the data to some grid that you specify. Besides the EzGet subroutines discussed in example 1, the following subroutine will be called:

defregrd .... This subroutine controls mapping of data to a specified grid.

Suppose we want to retrieve data and regrid it to a $10^{\circ}$ by $15^{\circ}$ latitude-longitude grid. The following program accomplishes this task.

program regrding 
This program

** retrieves global surface temperature data and maps it to a 10 degree by 15 degree latitude-longitude "target" grid using an area-weighted averaging scheme.

** creates an array of "weights", with elements set proportional to the sum of the areas of the original (i.e., source) grid cells that contribute to each target cell.

** obtains the longitude and latitude coordinates of the target grid and the length of each dimension of the target grid.

c This program is in many ways similar to example 1 , and

c further explanation can be found in the comments appearing in

c that example (program extract).

Note that in order to apply the area-weighting regridding algorithm, the user must specify what type of grid the original data were

c stored on (e.g., gaussian, evenly-spaced, etc.)

c The size of the arrays, adata and wtsmask, only need to be large c enough to accommodate the region extracted (i.e. after regridding), c so nlon .ge. $360 / 15=24$ and nlat $\cdot$ ge. $180 / 10=18$.

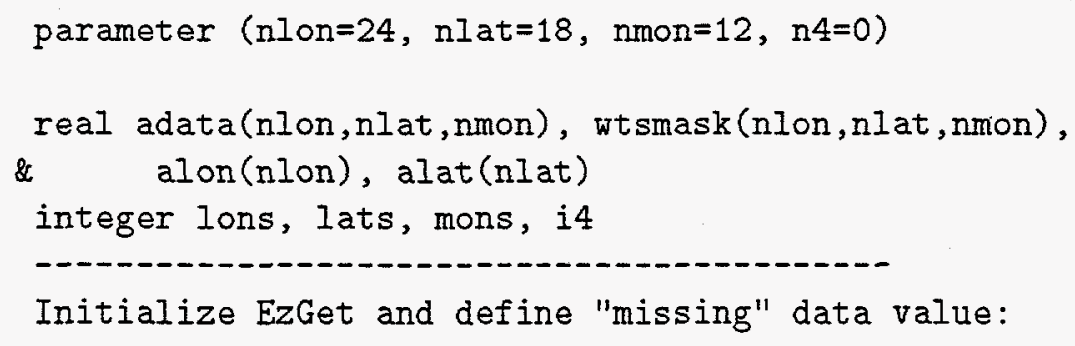

Define variable 1 as 'tas' and indicate path/filename where it is stored.

call defvar(1, 'tas', '/scratch/staff/lisa/amip_obs/nasa-amip_t') 
call defdim(1, 1, 'Iongitude', 'width', 'range', $0.0,0.0,360.0$ )

call defdim(1, 2, 'Iatitude', 'cosine', 'range', $0.0,0.0,0.0$ )

call defdim(1, 3, 'time', 'unit', 'nearest', 1.0,12.0,0.0)

C

C

c

c

c

c

c

c

c

$c$

$c$

c

c

c

c

$c$
When regridding data, the "cycle" for longitude should always be specified (set to 360.0 in the above example).

The domain limits specified for longitude and latitude will be overridden by the arguments in the call to subroutine defregrd below.

Define target grid to which data should be mapped. \&

call defregrd(1, 'uniform', 0, 'area-weighted', 18, 85.0, -10.0, where the arguments in the subroutine call indicate the following:

1: The first argument has the value 1 and indicates that the the regridding will be applied to variable 1 .

'uniform': indicates that the target grid will be rectangular grid of evenly-spaced latitude and longitude cells.

0 : This argument is ignored because the 2nd argument was set to 'uniform'.

'area-weighted': indicates that an area-weighted mapping scheme should be used.

$18,85.0,-10.0$ :

These 3 arguments define the latitude grid that will be created. In this case a grid with 18 latitude cells is created with the first grid cell centered at 85.0 degrees north and proceeding southward in increments of 10 degrees.

$24,-172.5,15.0$ :

These 3 arguments define the longitude grid, indicating that there will be 24 longitude grid cells, with the first grid cell centered at -172.5 degrees west and proceeding eastward in increments of 15 degrees.

Extract variable 1 from file and map to target grid defined above.

Ions $=24$

lats $=18$

mons $=12$ 


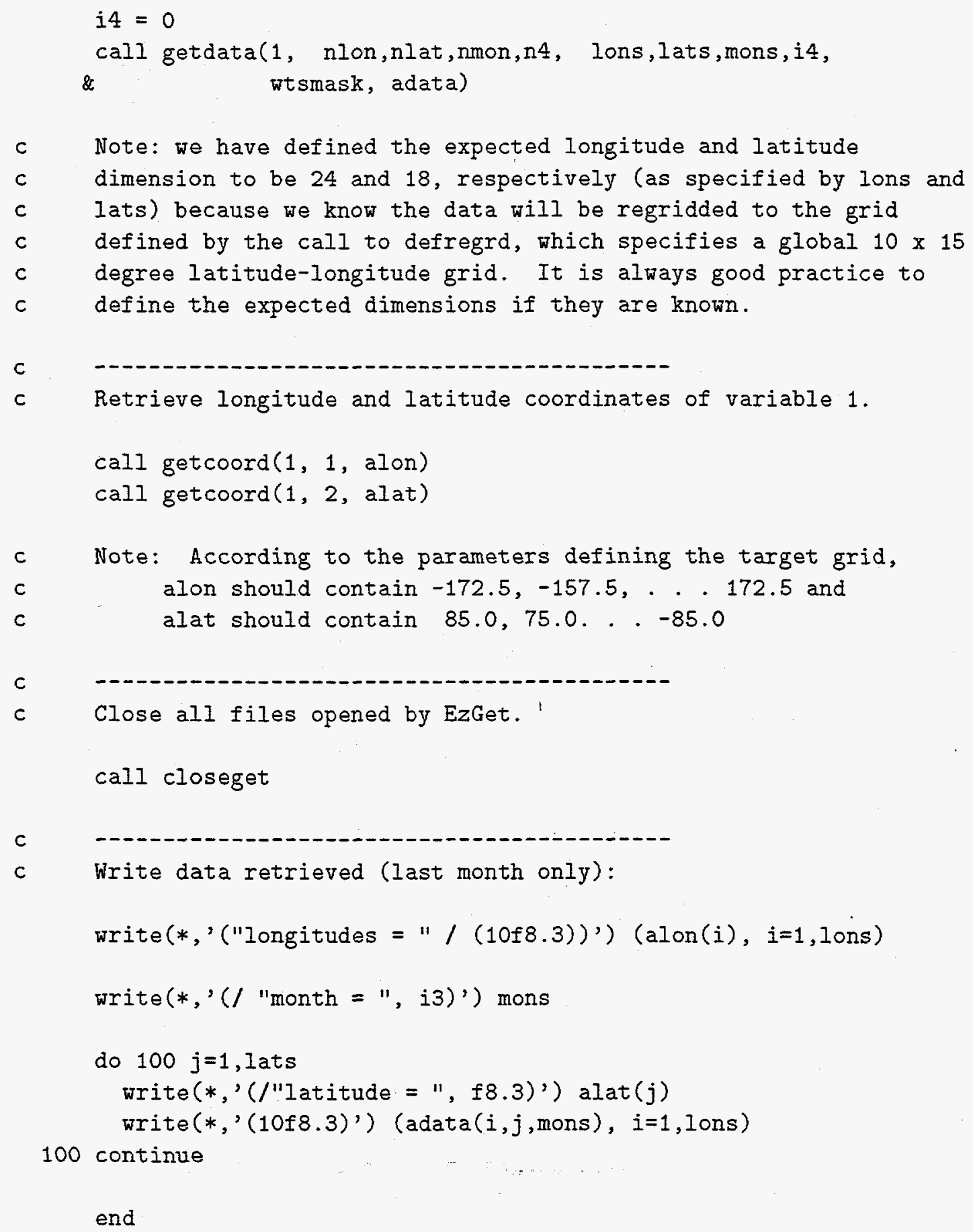




\subsection{Computing an area average}

There are several ways that EzGet can facilitate the computation of area averages. Suppose we want to compute the average temperature over North America north of the Tropic of Cancer for each month of data stored in a file. Perhaps the simplest method makes use of the area-weighting regridding algorithm and the masking capabilities of EzGet as illustrated by the following program:

program areamean

c This program

c ** retrieves surface temperature data for the region of

$c$

This program is in many ways similar to examples 1,2 and 3 , and

further explanation can be found in the comments appearing in

chose examples (programs extract, getregn, and regrding).

c $\gg$ Note that nlon and nlat can be declared as small as 1 , because

$c \gg$ these dimensions reduce to a single grid cell after regridding.

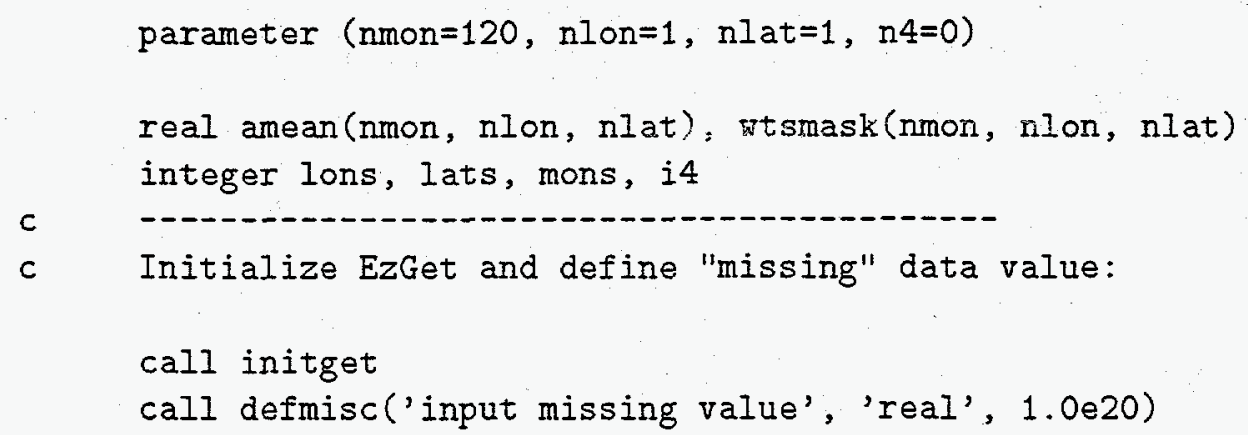


c Define variable 1 as observed surface temperature data ('tas')

c and define its domain:

call defvar(1, 'tas', '/scratch/staff/lisa/amip_obs/nasa-amip_t')

call defdim(1, 1, 'time', 'unit', 'as saved', $0.0,0.0,0.0$ )

call defdim(1, 2, 'longitude', 'width', 'range', $0.0,0.0,360.0$ )

call defdim(1, 3, 'latitude', 'cosine', 'range', $0.0,0.0,0.0$ )

c $\gg$ Note that in the 1st call to defdim above, the last 4

c $\gg$ arguments are:

c $\gg$ 'as saved', $0.0,0.0,0.0$ :

$c \quad \gg$ 'as saved' specifies that all months should be retrieved in

$c \quad \gg$ the order that they were originally stored in the file.

c $\gg$ (In this case the last 3 arguments $[0.0,0.0,0.0]$ are

c $\gg$ ignored.)

c

c Define variable 2 as the geography data needed and specify that

c it be used to select North American data only for variable 1:

call defvar(2, 'sftbyrgn', '/amipsp/drs/sftbyrgn/sftbyrgn_gla')

call defgeog(1, 'in', 2, 'North America')

c

c Define target grid to which data should be mapped.

$$
\begin{aligned}
& \text { call defregrd(1, 'uniform', } 0 \text {, 'area-weighted' } \\
& \& \quad 1,56.75,66.5,1,-125.0,150.0)
\end{aligned}
$$

c

c

c

c

c

c

where the target grid has been specified as a single grid cell centered at $125 \mathrm{~W}$ longitude, $56.75 \mathrm{~N}$ latitude and has latitude-longitude dimensions of $66.5 \times 150$.

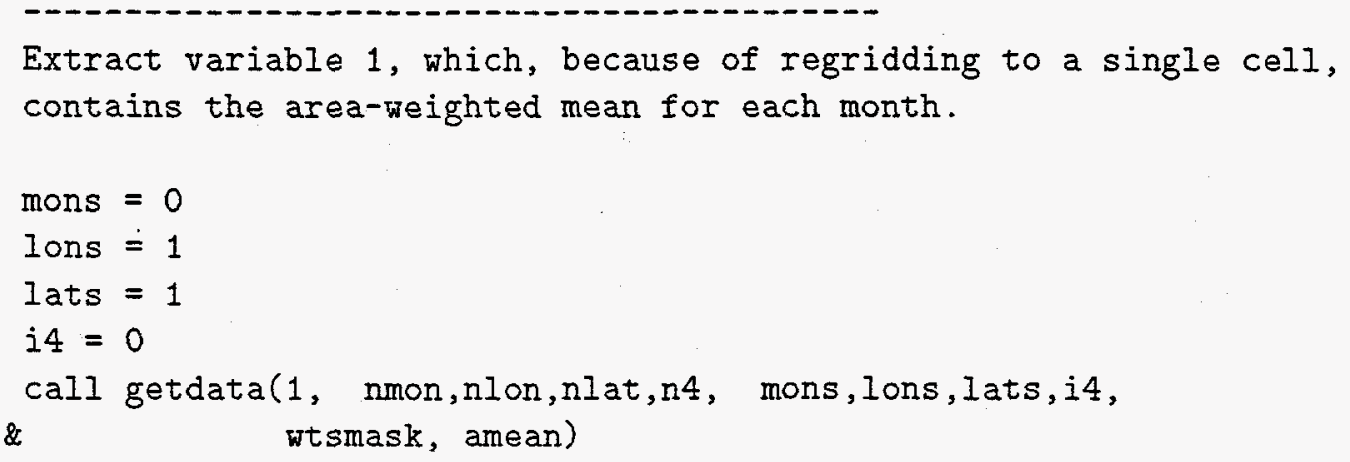




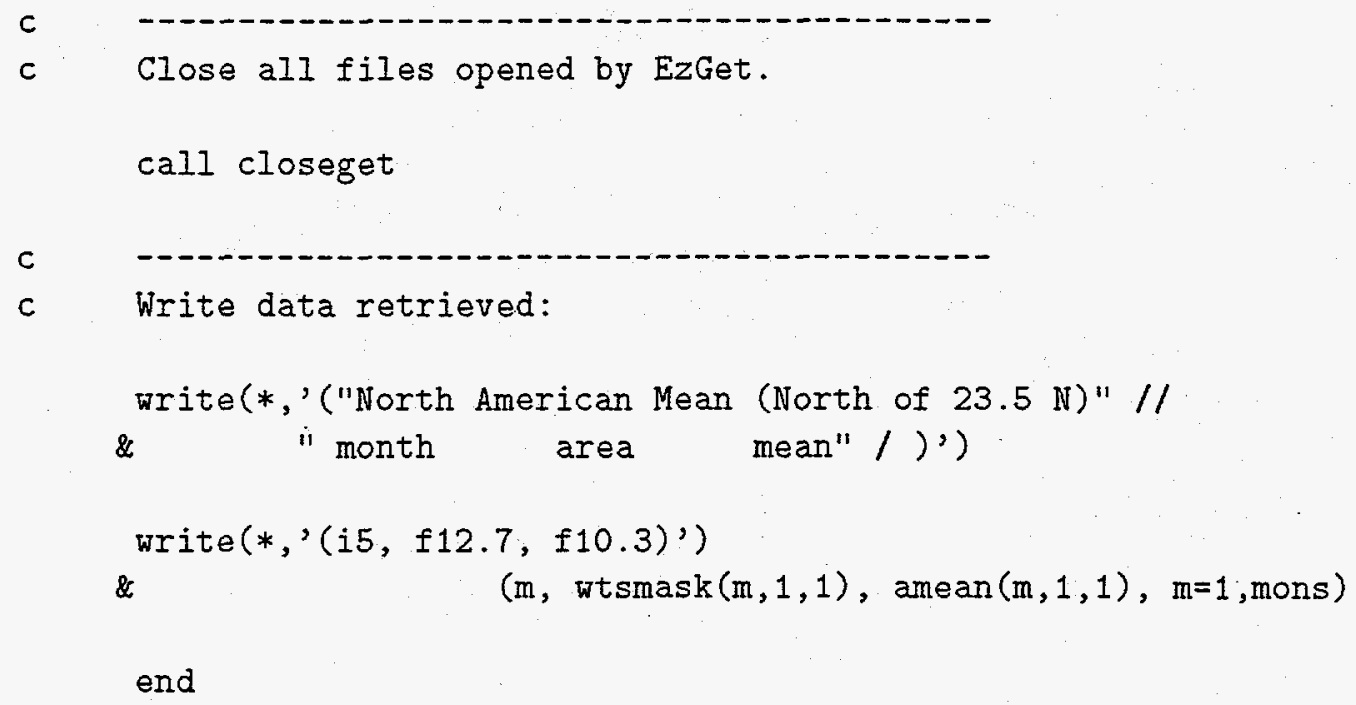

\subsection{A typical amip application}

Model output from the Atmospheric Model Intercomparison Project (AMIP) has been archived in a format readable by EzGet. It is often of interest to compare the model simulated fields by the AMIP models to observations. One measure of difference between two fields is the root-mean-square (RMS) difference. The following program computes the area-weighted RMS difference between model-simulated and observed fields of annual mean surface air temperature over North America in the year 1988 (i.e., year 10 of the AMIP simulations). Before computing the RMS difference, the model output is mapped to the same grid as the observations.

Note that it is not necessary for you to know either the resolution or the type of grid used by each model (because EzGet retrieves the resolution and for AMIP and PMIP models EzGet looks up the grid type in a table). 'The data extracted is mapped to the observed grid and all arrays you define are simply declared large enough to contain data on the observed grid. (EzGet dynamically allocates space needed to accommodate data on the original (i.e., source) grid.)

The following program will perform the RMS calculation:

$$
\text { program rmscalc }
$$

c This program loops through the AMIP models and computes the area-

$c$ weighted RMS difference between the modeled and observed annual mean

c surface air temperature over North America (north of the Tropic of 
c Cancer) for the year 1988. Only grid cells with observed values for

c every month of this year are included in computing the RMS

c difference. Because some models may have unrealistic

c representations of the continental geography, some data from the

c model's North American grid may not map onto the observed North

c American grid. Any grid cells where either observations or model

c data are missing are excluded from the RMS difference computed

c here.

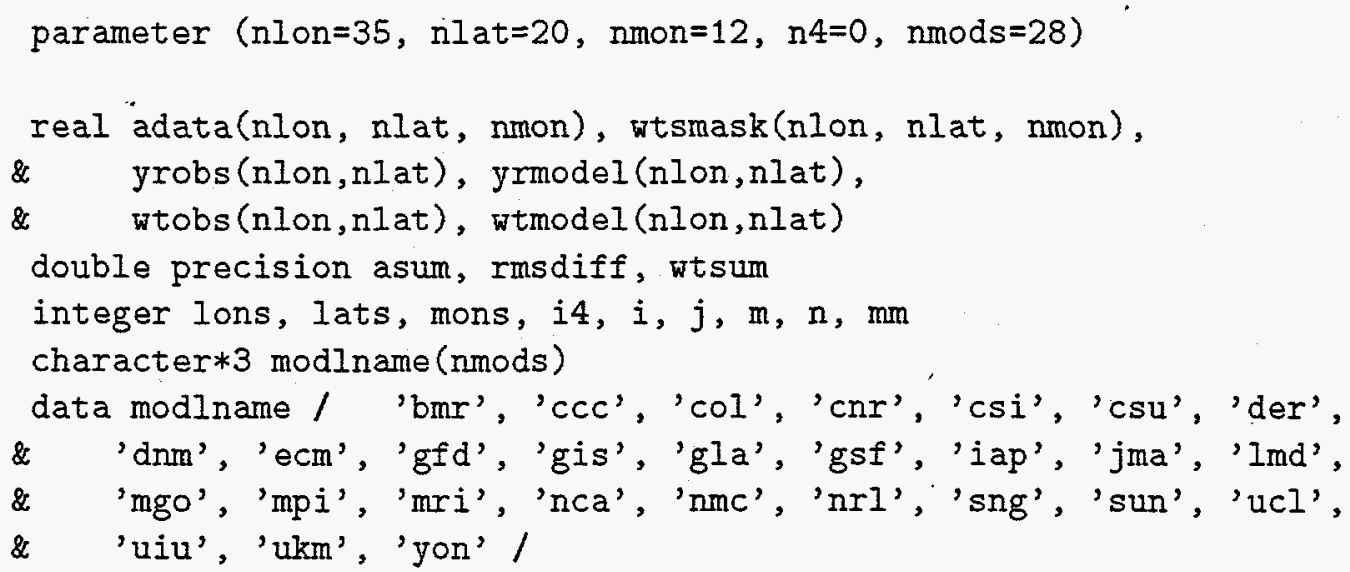

c Note: the 'rpn' and 'uga' models were left out of the above list

c because the variable 'tas' is not available for these

c models.

C

$c$

call initget

call defmisc('input missing value', 'real', 1.0e20)

C

c

c

Define variable 1 as the observed surface air temperature and indicate path/filename where data are stored.

call defvar(1, 'tas', '/scratch/staff/lisa/amip_obs/nasa-amip_t')

c

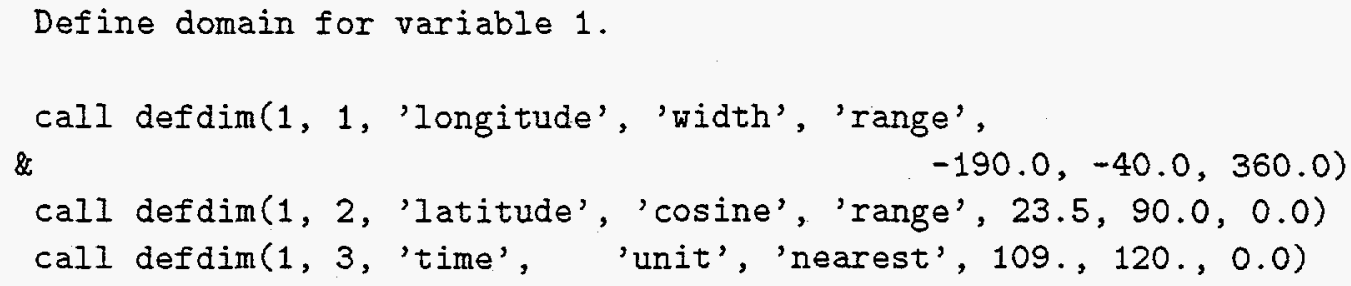


$c$

c

c

call defvar(2, 'sftbyrgn', '/amipsp/drs/sftbyrgn/sftbyrgn_gla')

call defgeog (1, 'in', 2, 'North America')

C

c Extract variable 1 (observed field).

lons $=0$

lats $=0$

mons $=$ nmon

$i 4=0$

call getdata(1, nlon,nlat, nmon,n4, lons,lats,mons, i4,

$\&$ wtsmask, adata)

c

c

c

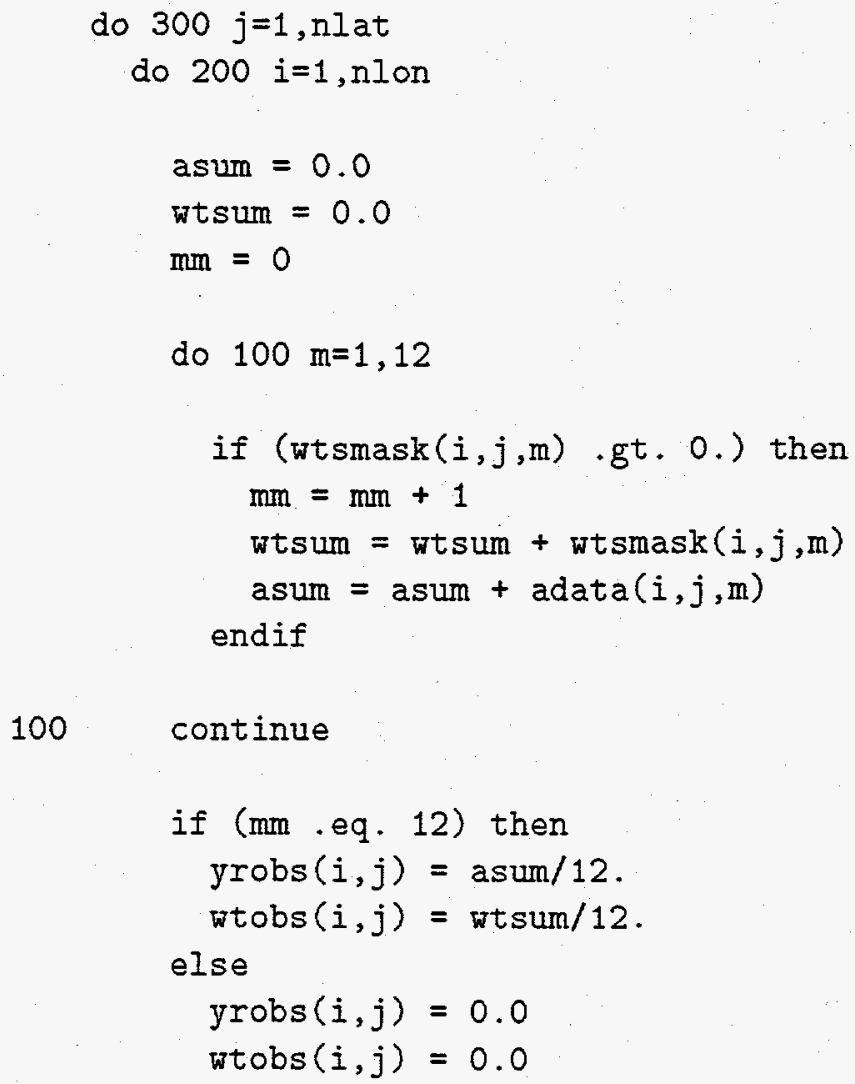


endif

200 continue

300 continue

c

$c$

c

Loóp through AMIP models and compute RMS differences between modeled and observed fields.

do $1000 \mathrm{n}=1$, nmods

c

c

c

c

c

$c$

c

c

c

c

$c$

c

c

$c$

c

Define variable 3 as the model simulated surface air temperature and specify the type of grid this model has:

call defvar(3, 'tas', '/amipsp/drs/tas/tas_'//modlname(n))

call defdim(3, 1, 'longitude', modlname(n), 'nearest',

\& $0.0,0.0,360.0$ )

call defdim(3, 2, 'Iatitude', modlname(n), 'nearest',

$\&$

$0.0,0.0,0.0)$

call defdim(3, 3, 'time', 'unit', 'nearest', 109., 120., 0.0)

\> Note that the domains specified above for latitude and

\> longitude are ignored by EzGet because the data for this

\> variable will be regridded to a target grid, which

\) determines the domain.

$\gg$ Note that the name of the model can be used to specify the

$\gg>$ type of longitude and latitude weights that will be

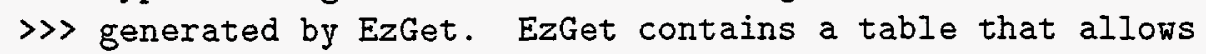

$\gg$ it to generate the proper weights proportional to grid cell

$\gg>$ area, if the model name is a standard AMIP or PMIP name.

\> Note that for AMIP data, months 109 through 120 are the

〉 months of the calendar year 1988 .

Define variable 4 as the geography data on the model grid, and specify that North American data should be selected for variable 3 .'

call defvar (4, 'sftbyrgn',

\&

'/amipsp/drs/sftbyrgn/sftbyrgn_'//modlname(n))

call defgeog(3, 'in', 4, 'North America') 
$c$

c

c \&

Instruct EzGet to map modeled field to observational grid upon retrieving data.

cảll defregrd(3, 'to', 1 , 'area-weighted',

$c$

c

\&

c

c

c

Extract variable 3 and map to observed grid.

lons $=0^{\circ}$

lats $=0$

mons $=$ nmon

i4 $=0$

call getdata(3, nlon, nlat, nmon, n4, lons, lats, mons, i4, wtsmask, adata)

Compute annual mean at each grid cell, but only if all 12 months of data are available.

do $600 j=1$, nlat

do $500 \quad i=1$, nlon

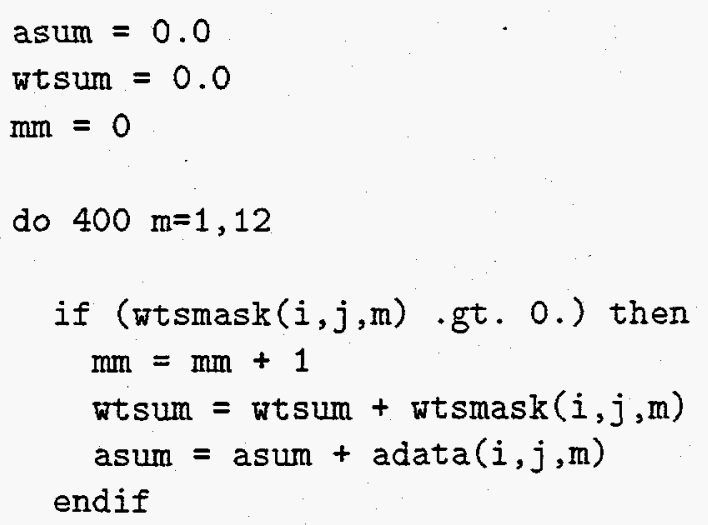

400

$$
\text { continue }
$$

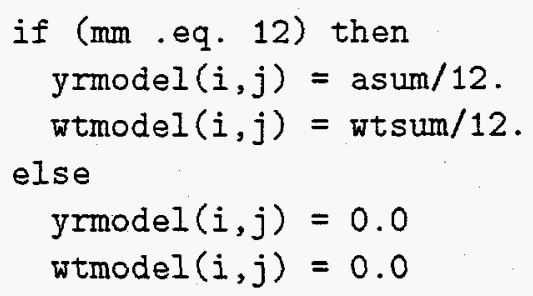




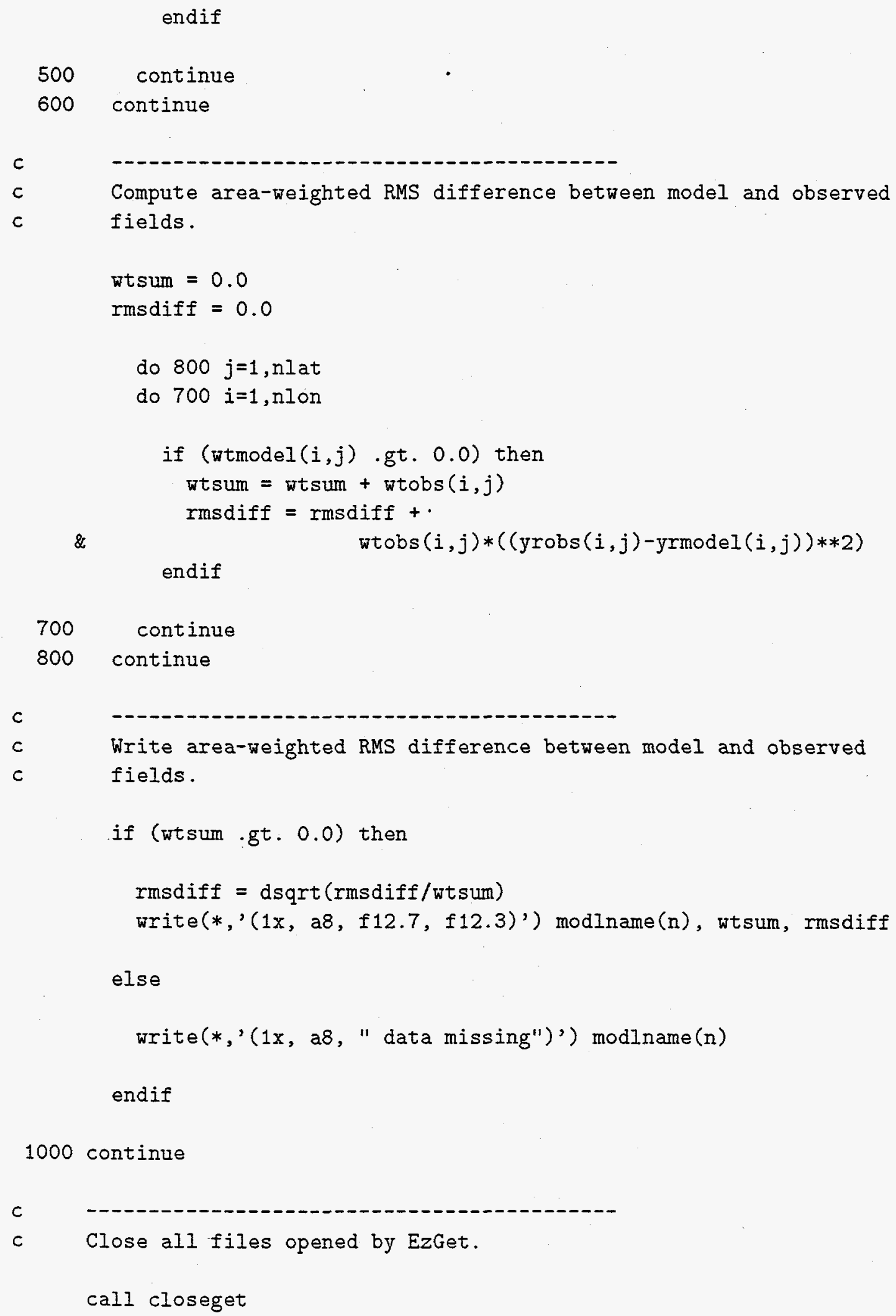


end

\subsection{Placing data in contiguous memory}

There are times when you might prefer that the data retrieved by EzGet be forced to occupy contiguous memory. Suppose, for example, that after you read the data, you plan to treat it as a vector string of elements (perhaps because vou do not care about the longitude-latitude structure of the data). Then instead of calling getdata, it may be more convenient to call getvdata, which always places the data retrieved by $\mathrm{EzG}$ et in contiguous memory. There is often a computational advantage to treating a single, one-dimensional vector of data rather than a multidimensional array containing the same data. Also it may be easier to reduce the internal memory required by EzGet if the data set is thought of as a 1-dimensional vector of elements.

In the following example, the maximum and minimum values of a field of unknown structure are found. By calling getvdata rather than getdata, we can make the program more general (while using memory efficiently). Besides the EzGet subroutines discussed in example 1, the following subroutines will be called:

getvdata .... This subroutine retrieves data and creates an appropriate mask (or set of "weights") associated with the data. It differs from getdata in that the data are forced to occupy contiguous memory.

domain .... This subroutine retrieves from a file the number of dimensions, the names of the dimensions and the full domain of each dimension of a field (as originally stored) for any variable.

lendims .... After retrieving data, this subroutine returns the actual length of each dimension of the data that has been retrieved.

program maxmin

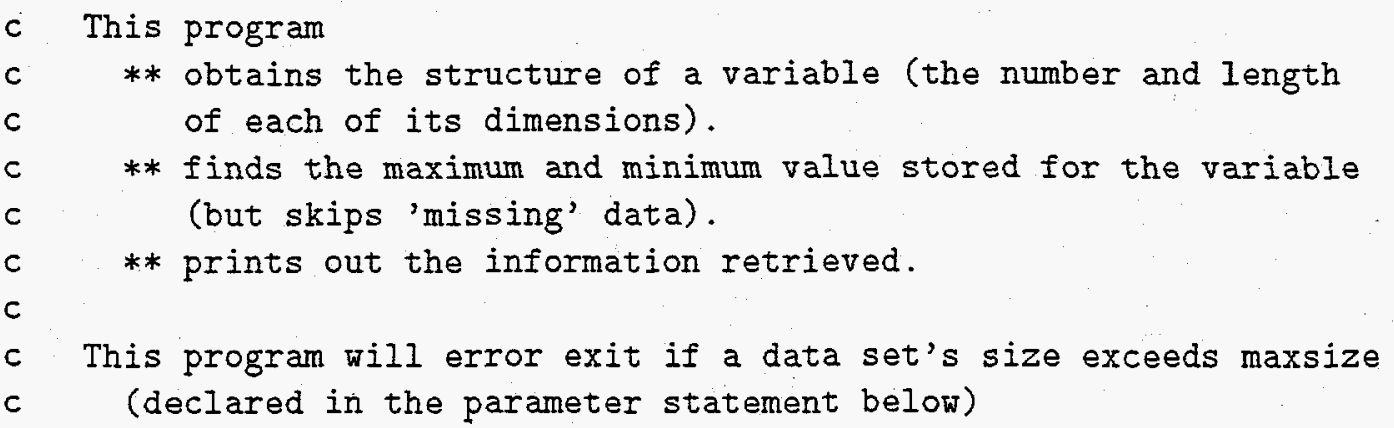




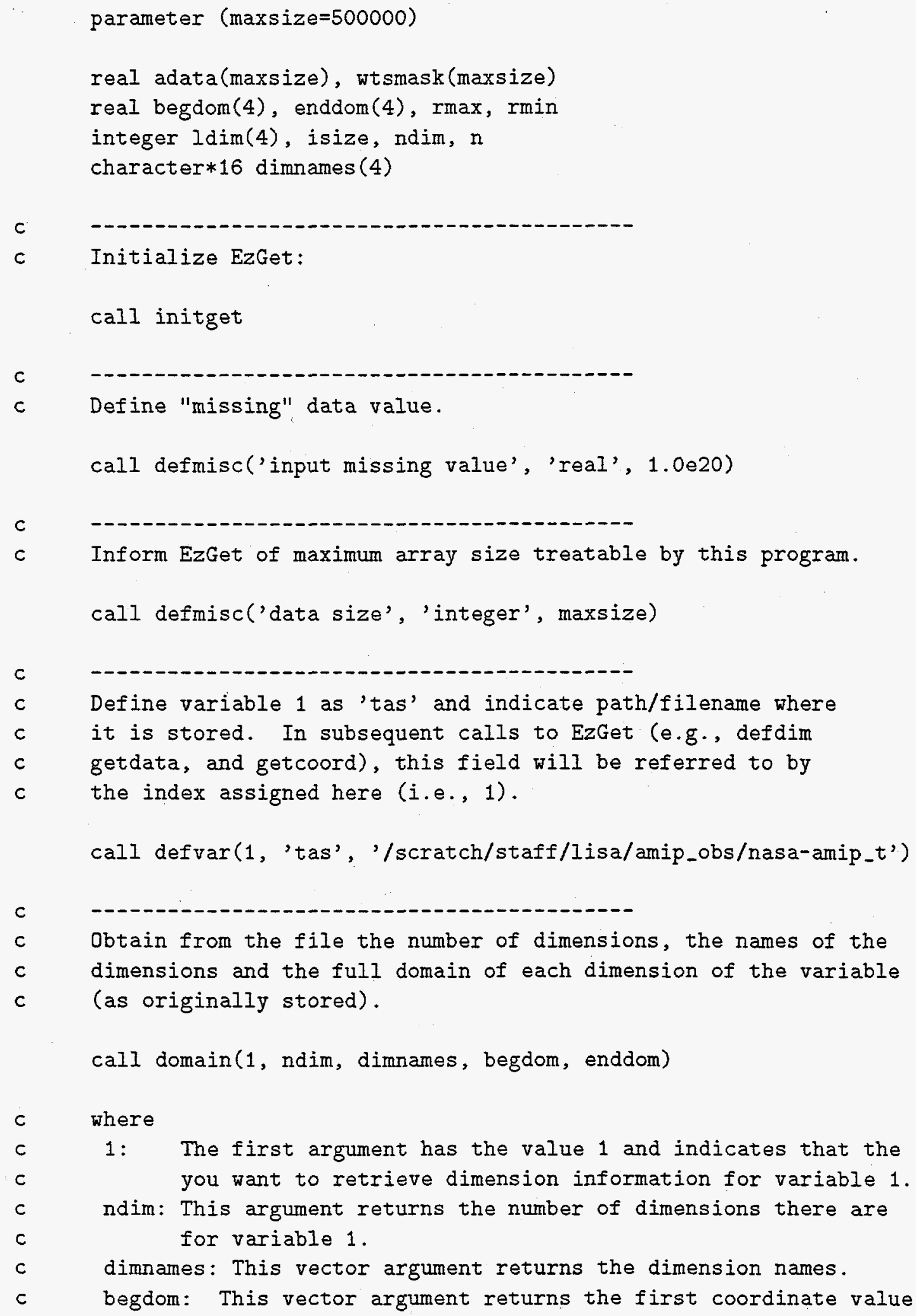

Obtain from the file the number of dimensions, the names of the dimensions and the full domain of each dimension of the variable (as originally stored).

call domain(1, ndim, dimnames, begdom, enddom)

c where

c 1: The first argument has the value 1 and indicates that the

c you want to retrieve dimension information for variable 1.

c ndim: This argument returns the number of dimensions there are

c for variable 1 .

c dimnames: This vector argument returns the dimension names.

c begdom: This vector argument returns the first coordinate value 


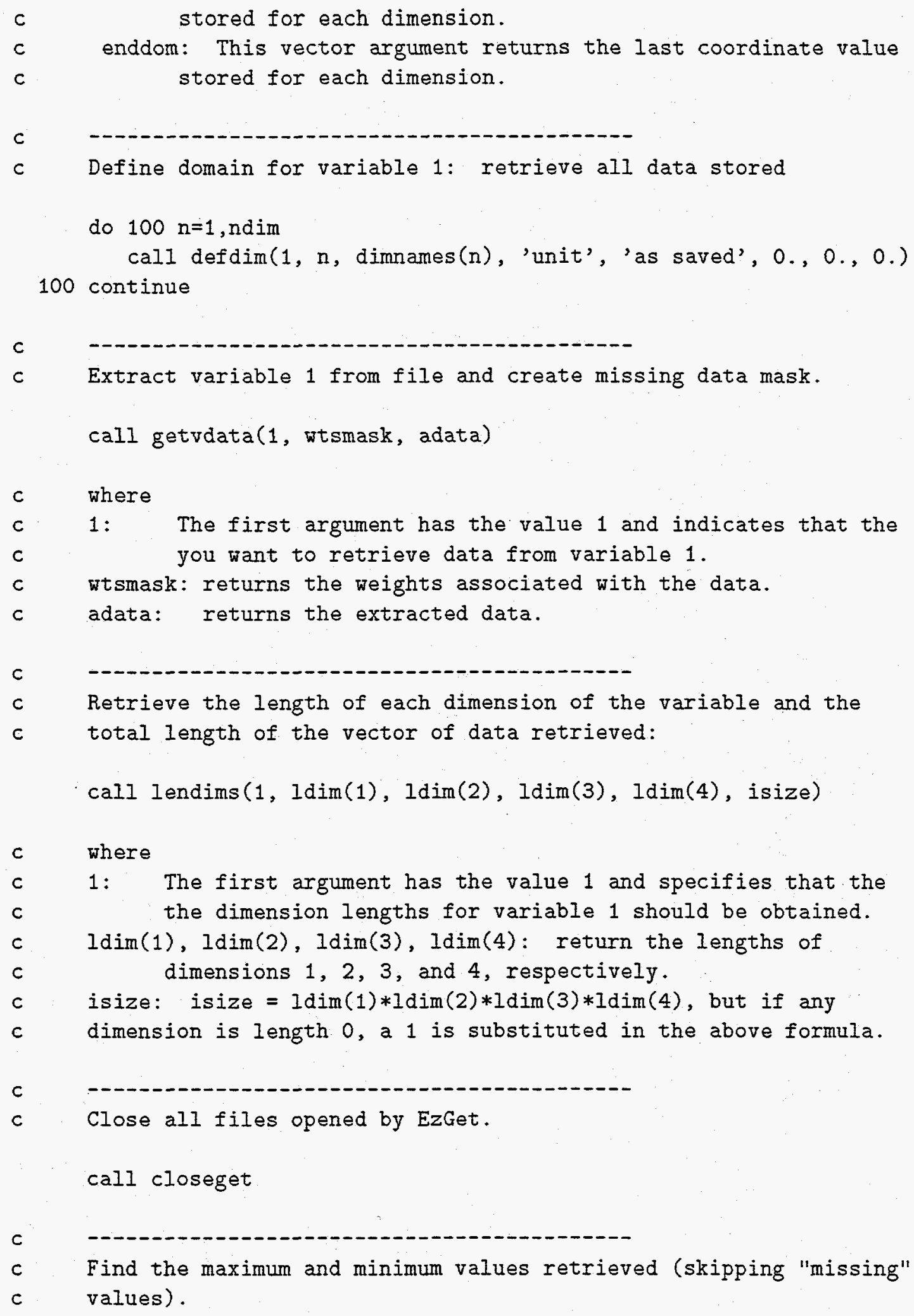




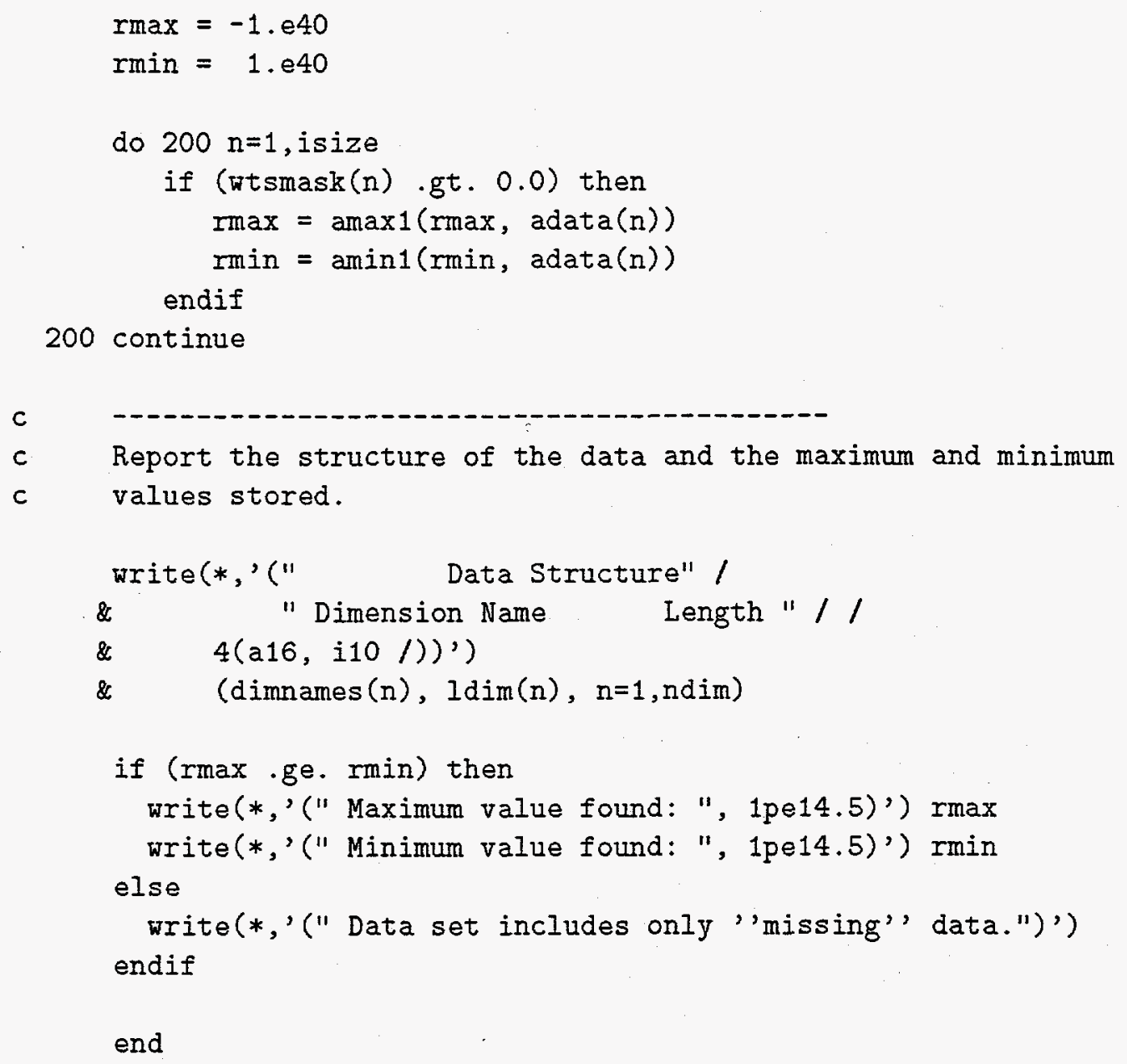

\subsection{Reducing memory requirements}

In the previous example the arrays might have been declared much larger than necessary, just to make the program general enough to retrieve the largest data set likely to be encountered. When memory is scarce, it would be better to declare the array to be just large enough to accomodate the data being retrieved. Furthermore, the data mask is not really needed in this case since missing values are also indicated by the value, $1.0 \mathrm{e} 20$, stored in array adata. The following revised version of the program given in the previous example dynamically allocates (upon execution) just enough memory to accomodate the data retrieved. It also avoids creating the data mask, which is not needed. Besides the EzGet subroutines discussed previously, the following subrontines will be called:

getfield .... This subroutine simply retrieves data. but does no masking or map- 
ping to a different grid. Unlike getvdata, no data mask or weights are returned. shape .... Before retrieving data, this subroutine returns the length of each dimension of a field as it will be retrieved by EzGet.

program smmaxmin

c This program

c ** obtains the structure of a variable (the number and length

c of each of its dimensions).

c ** finds the maximum and minimum value stored for the variable

c (but skips 'missing' data).

c ** prints out the information retrieved.

C

C

This program dynamically allocates enough memory to accomodate the retrieved data. It also avoids creating a data mask.

pointer (ptadata, adata)

real adata(*)

real begdom(4), enddom(4), rmax, rmin, err

integer $\operatorname{ldim}(4)$, isize, ndim, $n$

character*16 dimnames (4)

c

Initialize EzGet:

call initget

c

c

$\mathrm{c}$

c

Define "missing" data value. (Neither of these calls is actually necessary, since the default input and output missing value is $1.0 e 20$.

call defmisc('input missing value', 'real', 1.0e20)

call defmisc('output missing value', 'real', 1.0e20)

c

Define variable 1 as 'tas' and indicate path/filename where it is stored. In subsequent calls to EzGet (e.g., defdim

c getdata, and getcoord), this field will be referred to by

$c$ the index assigned here (i.e., 1).

call defvar(1, 'tas', '/scratch/staff/lisa/amip_obs/nasa-amip_t')

$c$ 
c Obtain from the file the number of dimensions, the names of the

c dimensions and the full domain of each dimension of the variable

c (as originally stored).

call domain(1, ndim, dimnames, begdom, enddom)

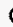

c Define domain for variable 1: retrieve all data stored

do $100 \mathrm{n}=1$, ndim

call defdim(1, $n$, dimnames $(n)$, 'unit', 'as saved', $0 ., 0 ., 0$.

100 continue

$c$

Retrieve the length of each dimension of the variable and the

c total length of the vector of data that will be extracted:

call shape(1, $\operatorname{ldim}(1), \operatorname{ldim}(2), \operatorname{ldim}(3), \operatorname{ldim}(4)$, isize)

c where

c 1: The first argument has the value 1 and specifies that

c the dimension lengths that will be returned for variable 1 are being requested. $\operatorname{ldim}(1), \operatorname{ldim}(2)$, etc.: return the lengths of dimensions $1,2,3$, and 4 , respectively.

isize: returns the size of the array needed to accomodate the retrieved data (which may be different from the number of elements stored in the file for this variable).

$c$

Allocate memory for array.

Note: some platforms may have slightly different functions for allocating memory dynamically.

ptadata $=\operatorname{malloc}($ isize $* 4)$

c where

c ptadata: is a pointer to array adata as declared at the beginning

c of this program.

c isize: specifies how many words to allocate for

c array adata.

$c$

c Inform EzGet of array size. 
call defmisc('data size', 'integer', isize)

c

c Extract variable 1 from file.

call getfield(1, adata)

c where

c 1: The first argument specifes that data should be retrieved

c from variable 1.

c adata: returns the extracted data.

C

c Close all files opened by EzGet.

call closeget

$c$

c Find the maximum and minimum values retrieved (skipping "missing"

c values).

$\operatorname{rmax}=-1 \cdot e 40$

$\operatorname{rmin}=1 . e 40$

do $200 \mathrm{n}=1$, isize

if (abs (adata $(n)-1.0 e 20)$.gt. 1.e15) then

$r \operatorname{rmax}=\operatorname{amax} 1(r \operatorname{rax}, \operatorname{adata}(n))$

$\operatorname{rmin}=\operatorname{amin} 1(\operatorname{rmin}, \operatorname{adata}(n))$

endif

200 continue

C

c Release memory allocated for adata.

2

$c$

Note: some platforms may have slightly different functions

c for releasing memory.

err $=f r e e($ ptadata $)$

c. where

c ptadata: is the pointer to array adata, as declared at the

c beginning of this program.

c

c Report the structure of the data and the maximum and minimum

c values stored. 


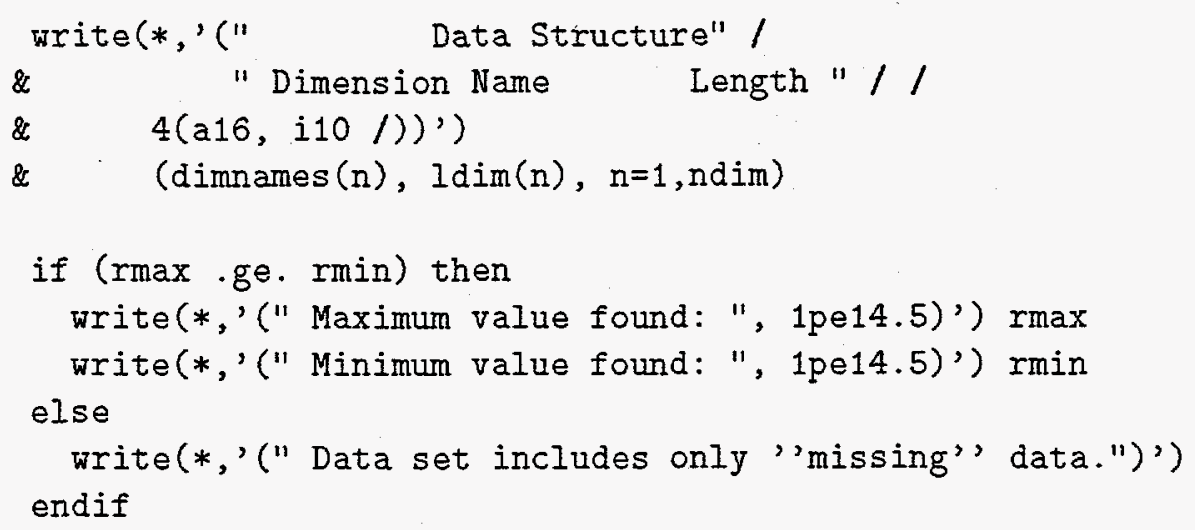

end

\subsection{Another example of area-averaging}

One of the advantages of retrieving data with EzGet is that an array of weights can be generated proportional to the area of the grid cells (and in the following example also proportional to the number of days in each month). This makes it easy to compute annual averages and area averages. Suppose, for example, you want to compute the global mean difference between modeled and observed annual mean temperature. The array of weights can be used to select only those regions of the globe where data are available for every month of the year. Then the same weights can be used to weight the data appropriately.

There is more than one way to proceed, but in the following program the model output is mapped to the grid of the observations before masking out regions of missing data.

program meandiff

c

c

c ** retrieves observed and model-simulated monthly surface air

c temperature for the year 1979 (first year of AMIP run).

c ** determines which grid cells are not missing any data and then

c for these grid cells

c ** computes the global average, annual mean difference between the

c observed and model-simulated temperatures. 
call defvar(2, 'tas', '/amipsp/drs/tas/tas_bmr')

c

c Define domain for variable 2 .

call defdim(2, 1, 'longitude', 'width', 'range',

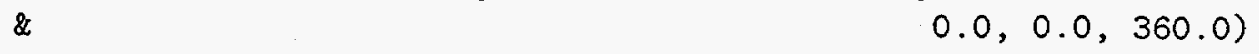

C

Compute annual mean at each grid cell, but only if all 12 months of data are available for both the model and the observations.

If data is available, weight by the area of the grid cell and the number of days in a month.

do $300 j=1$, lats
do $200 i=1$, lons

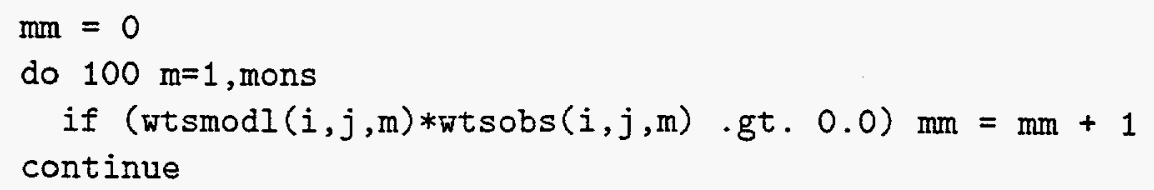


300 continue

c

c Compute global average, annual mean difference

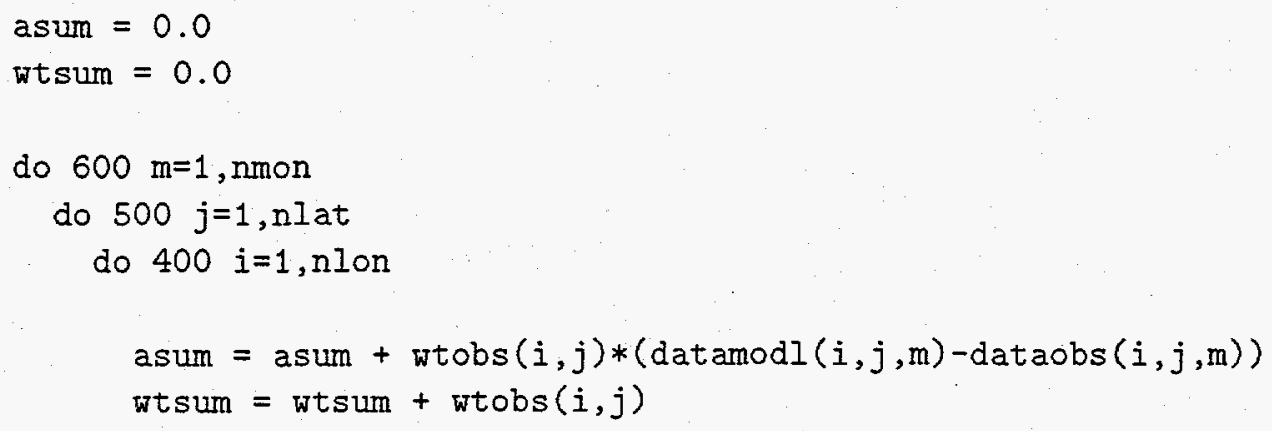

C

c Write area-weighted annual mean difference between model and

c observed fields.

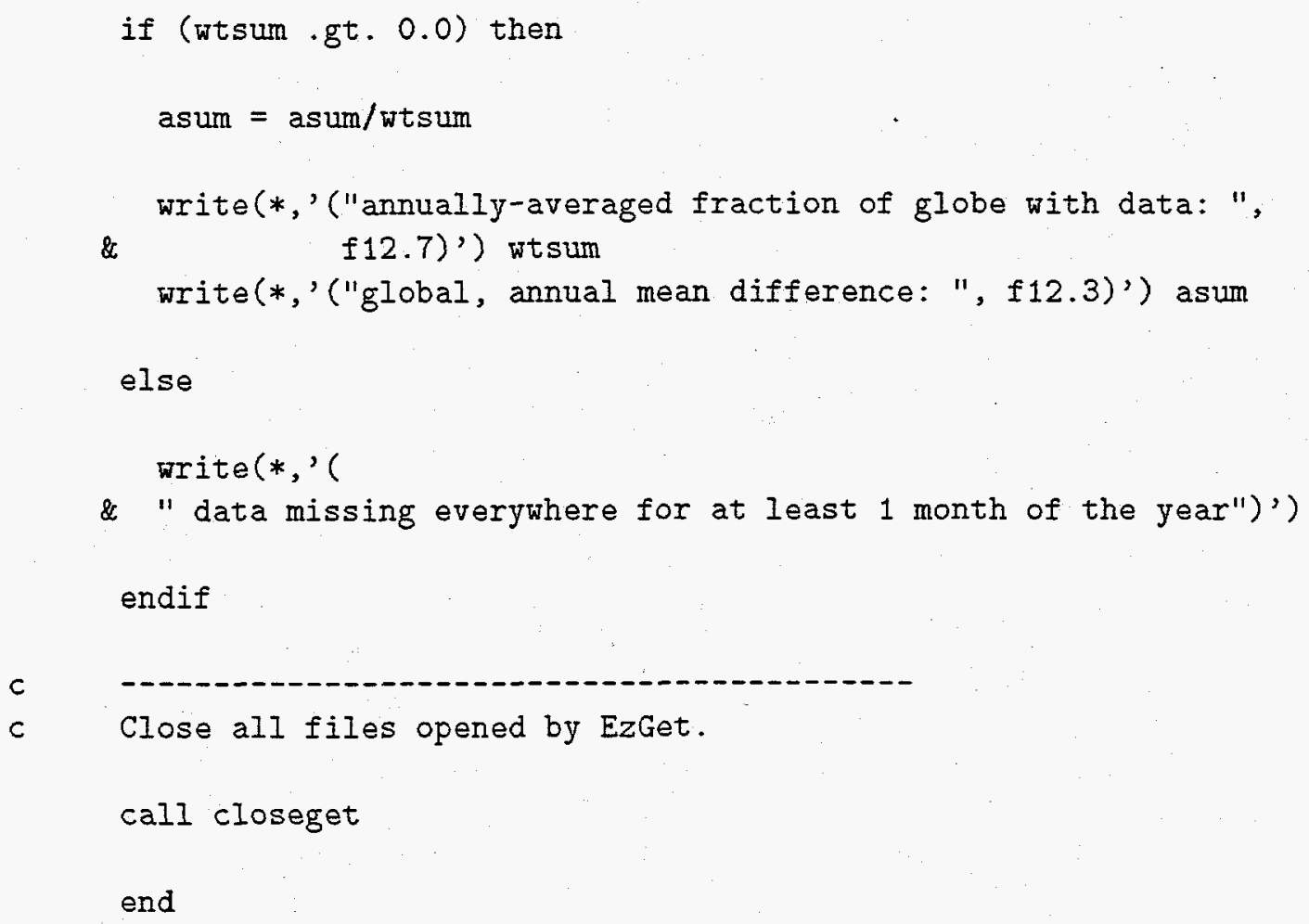




\section{$3 \quad$ EzGet Subroutines}

The EzGet subroutines that you will most likely need to call are documented in this section. First brief descriptions of the alphabetically ordered EzGet subroutines are provided:

closeget .... This subroutine closes any files opened by EzGet.

clrtable .... This subroutine clears a "dimension table" internal to EzGet. This may be necessary from time to time to make room for new dimension information.

defdim .... This subroutine specifies both the dimension ordering for data that will be retrieved and the desired domain. It also provides information for creating an array of "weights," typically set proportional to the grid-cell areas.

defdimi .... This subroutine is similar to defdim, but the domain is specified by index, rather than by coordinate range.

defgeog .... This subroutine is called to select data from certain geographical regions, so that when a field is retrieved, all data outside those regions are masked out.

defmisc ... This subroutine ('define miscellaneous') can be used to override certain default parameters assumed by EzGet (e.g., the value used to identify missing data, a parameter controlling the reporting of EzGet error messages, etc.).

defregrd .... This subroutine controls mapping of data to a specified grid.

defvar .... This subroutine defines a variable that will be referenced subsequently by a simple integer index.

defvarex .... This subroutine is similar to defvar but must be used if more than one variable with the same name is stored in a file.

domain .... This subroutine retrieves from a file the number of climensions, the names of the dimensions and the full domain of each dimension of a field (as originally stored) for any variable.

domlimit .... After data have been retrieved (or after shape has been called), this subroutine returns the domain limits of a specified dimension (which are determined by your specifications through calls to defdim).

getcoord .... After data have been retrieved (or after shape has been called), this subroutine returns a vector of coordinate values of a specified dimension.

getdata .... This subroutine retrieves data. possibly mapping it to a different grid and masking user-specified geographical regions, and creates an appropriate mask (or set of "weights") associated with the data. It differs from getvdata in that the data are put into a multidimensional array structure and do not necessarily occupy contiguous memory. 
getdimwt .... After clata have been retrieved (or after shape has been called), this subroutine returns a vector containing the weights associated with a specified dimension.

getedges .... After data have been retrieved (or after shape has been called), this subroutine returns a vector containing the locations of the grid cell edges (i.e., grid cell boundaries) for a specified dimension.

getfield .... This subroutine simply retrieves data, but does no masking or mapping to a different grid.

getgeog .... This subroutine creates a geography mask for a specified region. Normally you do not need to create this mask as a separate step because defgeog will already have made it possible to select the desired geographical regions.

getvdata .... This subroutine retrieves data, possibly mapping it to a different grid and masking user-specified geographical regions, and creates an appropriate mask (or set of "weights") associated with the data. It differs from getdata in that the data are forced to occupy contiguous memory.

initget .... This subroutine must be called to initialize EzGet. It assigns default values to a few parameters and sets up some internal tables.

lendims .... After retrieving data, this subroutine returns the length of each dimension of a variable that has been retrieved by EzGet. It would typically be called after retrieving data with getvdata or getfield.

shape .... Before retrieving data, this subroutine returns the length of each dimension of a field as it will be retrieved by EzGet.

varinfo .... This subroutine returns descriptive information retrievable from the file containing a defined variable. Information retrievable includes the data source, title, units, date, time, and rariable-type.

\subsection{Subroutine closeget}

This subroutine closes any files opened by EzGet. It is generally a good idea to call this subroutine before a program ends. Subroutine closeget has no arguments, so it is called as follows:

call closeget

Note that while EzGet is active (i.e.. after initget is called and before closeget is called), unit numbers 90-94 and 95-96 are reserved for EzGiet. (See Section 4.1 for further information.) 


\section{$3.2 \quad$ Subroutine clrtable}

This subroutine clears a "dimension table" internal to EzGet. The dimension table may become filled after too many fields with different coordinate dimensions have been retrieved by EzGet, and it may be necessary to make room for new dimension information. ${ }^{2}$ You will learn of this necessity by an explicit error message transmitted by EzGet, so you may choose to wait for such a message before including a call to clrtable. Subroutine clrtable has no arguments, so it is called as follows:

call clrtable

Once the dimension table has been cleared, it will be impossible to obtain the information about a variable previously accessed by EzGet, which would normally be retrievable by subroutines domlimit, lendims, getcoord, getedges, and getdimwt.

\subsection{Subroutine defdim}

This subroutine specifies the dimension ordering and the desired domain for data that will be retrieved. It also is called to specify information for creating an array of "weights," typically set proportional to the grid-cell areas. After creating the set of weights as specified by calls to defdim, EzGet may reset weights to zero where data are missing or have betn masked (e.g., if only a limited geographical region has been selected through a call to subroutine defgeog). A call to defdim is of the form:

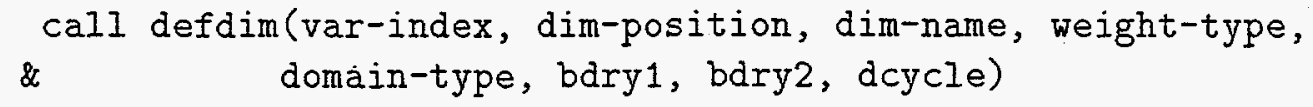

where

var-index (integer) specifies which variable (defined by calling defvar or defvarex) will be provided with dimension information by this call to defdim. If var-index is set to 0 , then the dimension information will be assigned to all variables.

dim-position (integer) specifies which dimension will be defined by this call to defdim. When the data are retrieved, the ordering of the dimensions is determined by this number. For example, if dim-position has a value of 2 , then the data will be retrieved such that this is the second dimension. (Note that. following the FORTRAN convention. the first dimension varies most rapidly as we move through contiguous storage.) When EzGet is initialized, all dimensions

\footnotetext{
${ }^{2}$ The dimension table contains room for 100 different coordinate dimensions, so it normally will become filled only when processing many different models.
} 
are designated as being 'not defined'. You may want to reset all dimensions to their "not defined" state, which can be done by calling defdim with dim-position set to 0 . You may also 'undefine' a single dimension by setting dim-position to a negative value. For example if dim-position is assigned a value of -2 , then the second dimension will be reset to 'not defined'. When dim-position is assigned $0,-1,-2,-3$, or -4 , the other arguments in the subroutine call are ignored (except, of course, var-index).

dim-name (character string) is the name of the dimension being defined.

weight-type (character string) controls the creation of the weighting-factor associated with the dimension. The total weight for a grid cell is the product of the weighting-factors for each of the grid-cell's dimensions. If data are missing or masked out, then the corresponding weights are set to 0 . The following options are available for this parameter:

'unit' - weight by 1 each grid-cell within the domain retrieved. This differs from 'equal' in that here the sum of the weights is equal to the number of grid-points in the retrieved domain of the dimension (before masking).

'equal' - weight equally each grid-cell within the domain retrieved. This differs from 'unit' in that here the sum of the weights over the full stored domain is equal to 1 (before masking). If only a fraction of the full domain is retrieved, then the sum of the weights will equal this fraction.

'width' - Set weights proportional to the width of each grid-cell, assuming the grid cells are equally spaced (i.e., assuming the grid-cell boundaries are half-way between the grid-cell centers). (Note, however, the edge of a pressure dimension is assumed to be greater than or equal to 0.) The weights generated are normalized by the total width of the domain that was originally stored. If a fraction of that domain is retrieved, then the sum of the weights will equal that fraction. (For a longitude dimension, the normalization is carried out under the assumption that the stored data spans $360^{\circ}$, even when it does not.)

'bmr', 'ccc', 'col', etc. - can be specified only for longitude or latitude dimensions and will create appropriate weights proportional to grid cell area for each of the AMIP or PMIP models. See Appendix A for a table identifying the model acronyms recognized by EzGet.

'gaussian' - weight each grid-cell assuming the dimension represents gaussian latitudes. Note that in creating gaussian weights, EzGet assumes as a default that in the file containing the data to be retrieved, the number of latitude grid-points spans the globe. For T21 resolution, for example, the number of latitude grid points stored in the file should be 32. If, in fact, data from some nonglobal latitude domain have been stored, then you must specify that the data have been stored on a subdomain of a T21 grid by calling subroutine defmisc as described in Section 3.6. 
'cosine' - weight by the cosine of latitude (i.e., $\mid \sin ($ edge1) - $\sin ($ edge2)|, where edge 1 and edge 2 are edges of a latitude grid-cell, assumed to be half-way between grid-cell centers).

'month' - weight by the number of days in a month. This assumes that the time dimension has units of months with January referenced by 1., 13., 25., etc., February referenced by 2., 14., 26., etc. The sum of the weights for a full year of data will be 1 . This weighting works correctly only for data from a 36.5-day year.

'leapyr' - weight by the number of days in a month. This assumes that the time dimension has units of months with January referenced by 1., 13., 25., etc., February referenced by 2., 14., 26., etc. The sum of the weights for a full year of data will be 1 . This weighting works correctly only for data from a 366 -day year.

domain-type (character string) is the argument determining the extent of the domain that will be retrieved. The following options are available for this parameter:

'as saved' - retrieve the full domain for this dimension in the same order that it appears in the file.

'range' - retrieve data from the domain specified by bdry 1 and bdry2. If the weighting option (weight-type) is specified as 'width', 'gaussian', 'cosine', or a model acronym (e.g., 'bmr', 'cce', etc.), then if any part of a grid cell falls within these boundaries, data will be retrieved from that grid cell (even if the actual coordinates of that grid cell lie outside the specified range). If the weighting option (weight-type) is specified as 'equal', 'unit', 'month' or 'leapyr', then only data with coordinates that lie within the domain specified by bdry 1 and bdry 2 will be retrieved. (Note that in this case, if you specify a narrow range for the domain, smaller than the width of a grid cell, it is possible that no grid-cell centers will lie within the domain, and no data will be retrieved.) Under all weighting options, if all grid cells lie completely outside the domain requested, then no data will be retrieved.

'nearest' - retrieve data with coordinates within the range specified by bdry1 and bdry2, except under the following two circumstances: 1) if no coordinates fall within bdry1 and bdry2, then retrieve the plane of data nearest to the region specified by bdry 1 and bdry 2 , or 2) if dcycle is not 0.0 and exactly one complete cycle is specified (i.e.. $\mid$ bdry $2-$ bdry $1 \mid=$ dcycle), then $E_{z}$ Get will shift the domain by up to $1 / 2$ grid-cell in order to avoid splitting the grid-cell at the edge of the domain. (Examples will be given later in this section.)

bdry1 (real) specifies the desired beginning boundary of the domain to be retrieved, but note that this argument is ignored if domain-type is set to 'as saved'. If bdry1>bdry2, then the order of data retrieval will be such that this coordinate 
decreases monotonically.

bdry2 (real) specifies the clesired ending boundary of the domain to be retrieved, but note that this argument is ignored if domain-type is set to 'as saved'.

dcycle (real) indicates the period of cyclic coordinates such as longitude (e.g., if the units are degrees longitude, then $-180.0=180.0=540.0$ and the cycle interval is 360.0 degrees) or possibly the annual cycle (e.g., if the units are months, then $1=$ $13=25$, and the cycle interval is 12 months). With this information, EzGet can find data that appear to lie outside of the range specified by bdry 1 and bdry2, but in fact are simply assigned a different but equivalent coordinate value. For non-cyclic coordinates, dcycle should be set to 0.0 . This argument is ignored if domain-type is set to 'as saved'. In all cases of cyclic data only a single cycle should reside on the file (i.e., do not include any "wrap-around points"). This implies that if multiple years of monthly data reside on the file, you should always set cycle to 0.0 , not 12.0 , for the time dimension.

The assignments made by a call to defdim remain in effect unless you subsequently call either defdim or defdimi and redefine dimension dim-position for variable var-index, or unless you reinitializes EzGet with a call to initget. If you instruct EzGet to map data to a new grid and if the call to subroutine defregrd specifies target-cntrl as 'to', then domain-type, bdry1, and bdry2 will be overridden and the domain will be that specified for the target grid (but which dimension varies most rapidly will still be determined by the defdim calls for the source data). If you instruct EzGet to map data to a new grid and if the call to subroutine defregrd specifies target-cntrl as 'uniform' and nlat>0 or nlon>0, then domain-type. bdry1, and bdry 2 will be overridden and the domain will be determined by the arguments in your call to defregrd.

At first you might be somewhat baffled as to what to specify for weight-type and domain-type, but the most usual choices are described here:

- Latitude: Usually you will want to extract a precisely defined domain (not necessarily coinciding with grid-cell boundaries) and create weights proportional to grid cell area. If this is the case then domain-type should be set to 'range' and weight-type should be set to either 'cosine' or 'gaussian', depending on the grid. In many cases a convenient way to generate area-weights is to set weight-type to the model acronym as given in Appendix A.

- Longitude: Usually you will want to extract a precisely defined domain (not necessarily coinciding with grid-cell boundaries) and create weights proportional to grid cell area. If this is the case then domain-type should be set to 'range' and weight-type should be set to either 'width' or the model acronym as given in Appendix A. If, however. you want to retrieve data spanning 360 degrees. but 
without possibly splitting one grid cell in two, then domain-type should be set to 'nearest'.

- Time (month): The most common choice for domain-type is probably 'nearest' when monthly data are retrieved. In order to create weights appropriate for computing annual means from monthly data, specify either 'month' or 'I eapyr', depending on whether the year is of normal length or a leap year. ${ }^{3}$ If, however, the model year comprises twelve 30 -day months (rather than the realistically defined calendar months), then one would normally specify 'equal'.

- Level or Time (hour, day. year, etc.): In this case the usual choice for domaintype will be 'nearest'. You will usually want to assign equal weighting to each grid cell, so set weight-type either to 'unit' or 'equal'. Note that pressure (i.e., mass) weighting cannot be generated by EzGet. Also note that if a dimension is very long (more than 20,000 elements in the file from which you are retrieving data or more than 2000 elements actually retrieved, then 'unit' weighting should be specified, unless the default lengths just quoted are overridden by a call to subroutine defmisc as described in Section 3.6.

If a given dimension of a variable (as it is stored) contains only 1 element, some of the above weighting factors will not work properly. For this special case, EzGet will override your specifications and assign this dimension 'unit' (except if 'month' or 'leapyr' has been specified, in which case the weight will be equal to the number of days in the month divided by the number of days in the year).

For further information concerning the difference between the various specifications of weight-type and domain-type consider the following. Suppose one dimension of an array of stored data has coordinates $3.0,4.0,5.0,6.0$, and 7.0 as illustrated below (with the boundaries of each grid cell indicated by vertical line segments and the coordinate positions indicated by the dots):

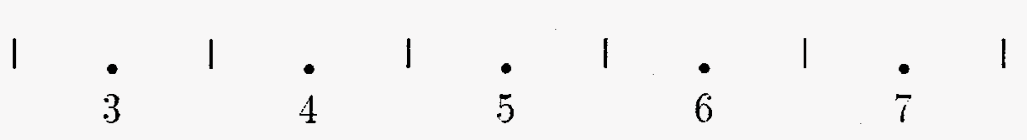

Unless 'gaussian', 'month', 'leapyr', 'csu', 'ucl', 'Imd', or 'Imd' has been specified as the weighting option. EzGet assumes that the boundaries of the grid-cells lie half-way between the coordinates (i.e.. at 2.5, 3.5, etc.). For this dimension suppose we want to extract a certain sub-domain of this data. The following examples indicate some options for controlling the domain that will be retrieved and the weights that will

\footnotetext{
${ }^{3}$ Note that this only works when the time dimension being defined is in units of months with month 1. 13, 25, etc. corresponding to January.
} 
be assigned to each cell containing data. In these examples we assume no missing data and no masking.

In the following illustrations the domain you request by calling defdim is indicated by the over-bracket and the extracted grid-points are indicated by the symbols, **'. The weights assigned each retrieved grid point are proportional to the intervals defined by the underbrackets. The illustrations show that the extracted grid-points and the weights depend on the values assigned to weight-type and domain-type:

\section{Example 1:}

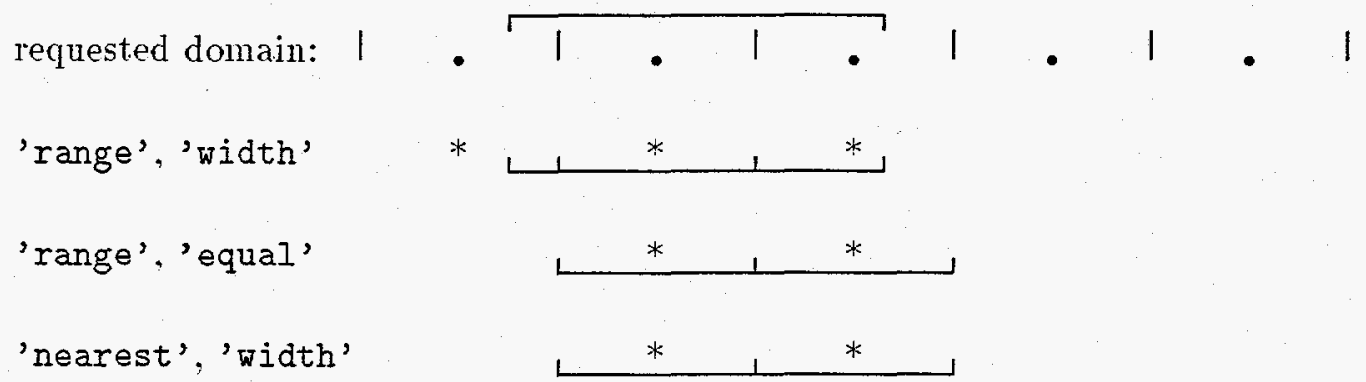

Example 2: No wrap-around permitted (i.e. you have specified dcycle to be 0.0 ).

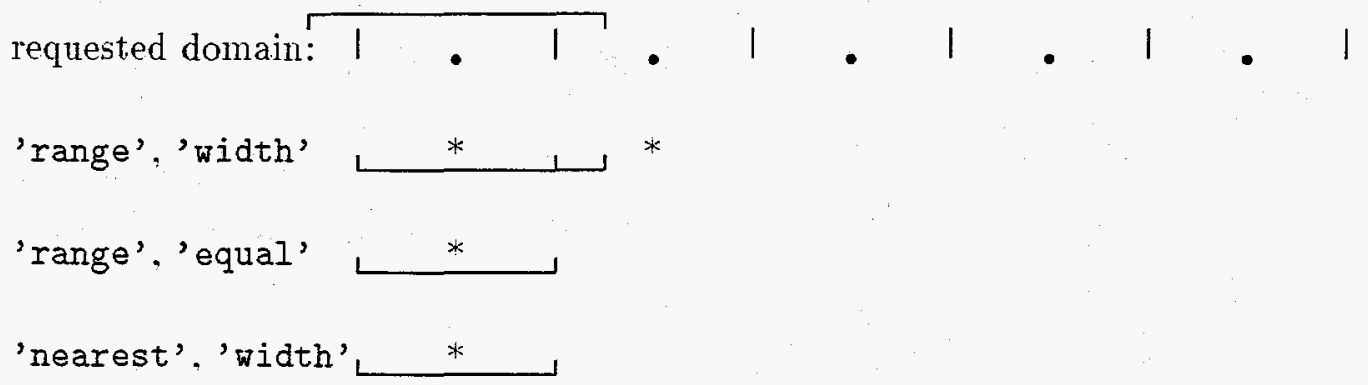

Example 3:

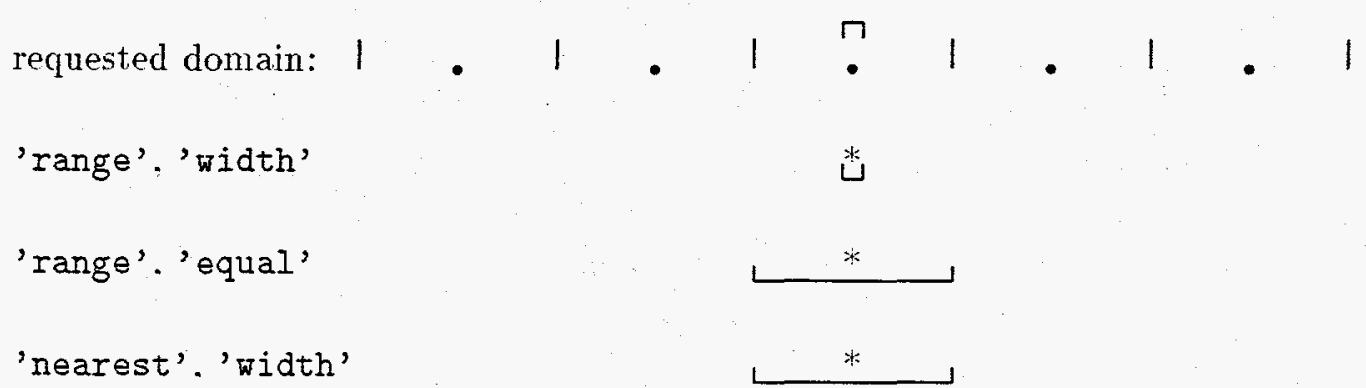


Example 4:

requested domain:

'range', 'width'

'range', 'equal'

no data retrieved

'nearest', 'width'

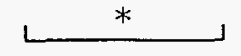

Example 5: No wrap-around permitted.

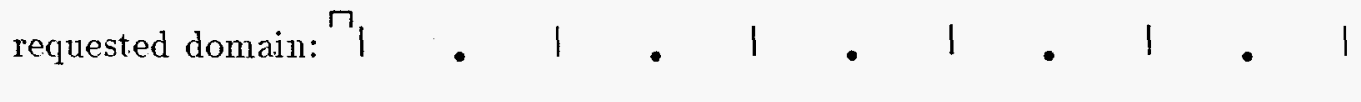

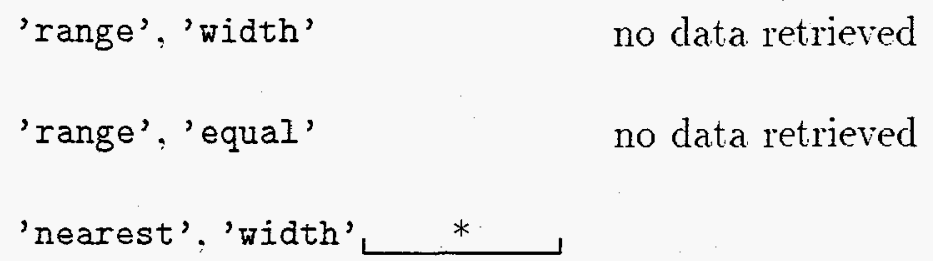

Suppose one dimension of an array of data is longitude with coordinates $-180 .,-170$, ..., 160., 170. as illustrated below:

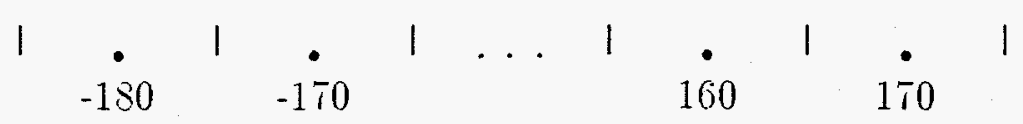

If the wrap-around option is in effect, then each grid-cell can be identified by more than one coordinate value. For example.

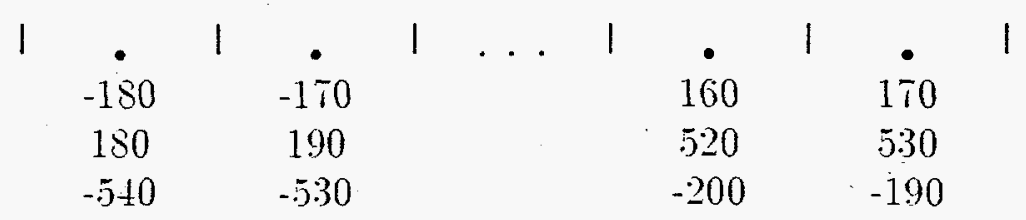

Example 6: Wrap-around activated (i.e., dcycle set to 360.0).

Suppose bdry 1 is assigned the value 162 . and bdry 2 is assigned the value 180. then the wrap-around of data implies the following (because the last grid cell extends only to 175 , the data is wrapped around. duplicating the first grid-cell in the last position): 

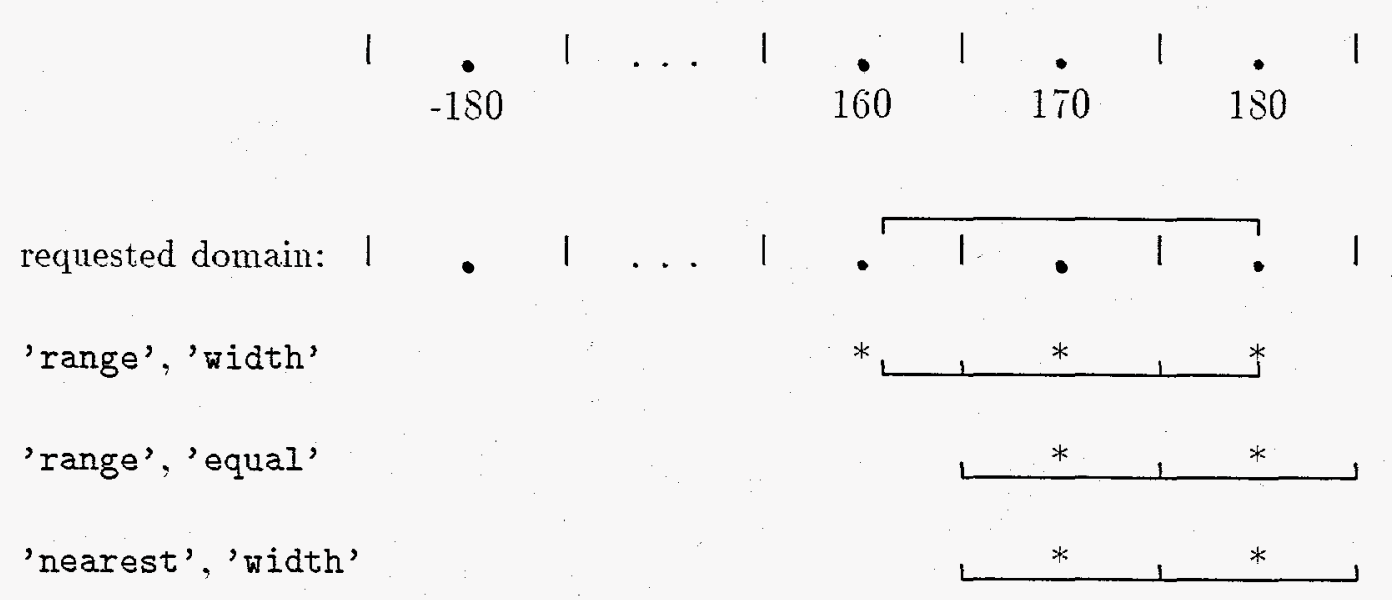

Example 7: Wrap-around activated, complete cycle requested.

Suppose bdry1 is assigned the value -180. and bdry 2 is assigned the value 180 ., then the wrap-around of data implies the following:
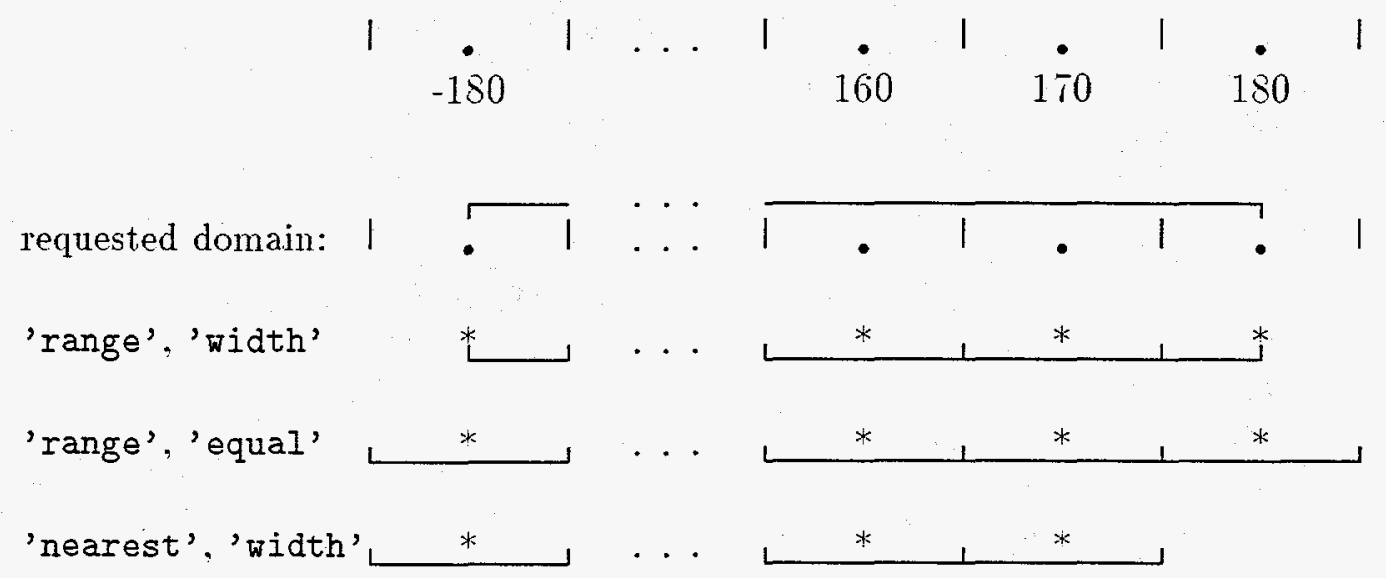

Note that when 'range' and 'width' are specified, data are extracted covering the full domain (from -180 to 180 ). which requires one grid cell to be split into two, each given half the weight of the original. To avoid splitting grid cells (and to extract a complete cycle of $360^{\circ}$ ), 'nearest' should be specified in which case the domain extracted is shifted slightly to the interval -185 to 175 .

\subsection{Subroutine defdimi}

This subroutine is similar to subroutine defdim but the domain is specified by index. 
rather than by a coordinate range. A call to defdimi is of the form:

call defdimi(var-index, dim-position, dim-name, weight-type, \& ind $x 1$, ind $x 2$ )

where

var-index, dim-position, dim-name, and weight-type are defined exactly as in subroutine defdim.

indx1, indx2 (integers) specify by index the first and last planes of data that should be retrieved. For example, if indx 1 and indx 2 are specified as 3 and 5 , respectively, then the third, fourth and fifth planes of dimension dim-name will be retrieved. If indx 1 and indx 2 are specified as 5 and 3, respectively, then the fifth, fourth and third planes of dimension dim-name will be retrieved (in that order). If ind $x 1$ and indx 2 are specified as -5 and -3 , respectively, then the fifth, fourth and third planes from the $\epsilon$. $d$ of the domain will be retrieved (i.e., $j j-4, j j-3$. and $j j-1$. where $j j$ is the length of the dimension. And as a final example, if indx 1 and indx 2 are specified as -3 and -5 , respectively, then the third, fourth and fifth planes from the $\epsilon$ d of the domain will be retrieved.

\subsection{Subroutine defgeog}

This subroutine is called in order to specify the geographical regions (within the domain defined by calls to defdim), from which data will be retrieved. All data outside the selected regions will be masked out (i.e., the "weights" will be set to 0.0 and the data will be set to the "missing value").

For selection of geographical regions, an input data set must be provided, which may either be a simple land-ocean-seaice mask (or even more simply, a land-sea mask) or a more detailed geographical data set uniquely defining regions typically the size of a continent (as described in more detail below). Appendix B contains the list of regions identified on the standard geographical maps for AMIP and PMIP models available from PCMDI. The grid on which the geography mask is stored must be identical to either the grid of the source data being retrieved or a new grid to which the data will be mapped.

A call to defgeog is of the form:

call defgeog(var-index, inorout, mask-index, select)

where 
var-index (integer) specifies to which variable (defined previously or subsequently by a call to defvar or defvarex) the geography mask will be applied.

inorout (character string) indicates whether the geography mask should be applied before or after mapping data to a new grid. This argument should be assigned one of the following strings:

'in' - if the geography mask should be applied before mapping data to a new grid.

'out' - if the geography mask should be applied after mapping data to a new grid.

mask-index (integer) specifies the variable (defined previously or subsequently by a call to defvar or defvarex) that contains the geography data.

select (character string) is a list of regions, separated by commas, specifying which geographical regions of the globe should be selected (i.e., not masked). If the geography data simply indicates regions of land, ocean, and possibly sea-ice, then this argument might be, for example, 'ocean, seaice', which would select all regions except land. If the geography data contains the more detailed geographical information, then this argument might be, for example, 'North America, South America, Greenland', which selects the major land areas of the Western Hemisphere. Note that the commas separating the listed regions may optionally be followed by blanks. For example, either 'Africa, Australia, Antarctica' or 'Africa, Australia, Antarctica' is acceptable.

Note that if you call degeog twice with inorout set to 'in' for one call and 'out' for the other call, then one geography mask can be applied before mapping to a new grid, and another mask can be applied after the mapping. The argument passed as select would usually be the same when selecting geography both before and after mapping to a new grid, but this is not required. You might, for example, select 'Iand' before mapping and 'North America' after mapping to the new grid.

Even when data remain on the original grid, two different masks can be applied by calling defgeog twice, once with 'in' specified and once with 'out' specified. You might want to do this, for example, in order to select ice free regions of the North Atlantic. You would call defgeog to select 'N. Atl. ocean' (available from 'sftbyrgn' variable) and then call defgeog a second time to eliminate regions of sea. (by selecting 'ocean' from the 'sft' variable).

If this subroutine is not called. then $\mathrm{E}_{z}$ Get will not select geographical regions (other than rectangular regions determined by the domain specifications). If after calling this subroutine, you want to return to this default state of no masking, defgeog should be called, but with mask-index set to 0 . If you had specified that masks be applied both before and after mapping the data to a new grid, then defgeog would have to be called 
twice with mask-index set to 0 , once with inorout set to 'in' and a second time with it set to 'out'. When mask-index is set to 0 , the last argument, select, is ignored.

Note that the geography must be at least a function of longitude and latitude. It mav also be a function of time (and perhaps in unusual circumstances, level). If it is a function of time (and if the time-dimension is greater than 1), then the time-dimension should be consistent with the time dimension for the data that you want to retrieve. If the geography field has fewer dimensions than the array of data, then the same geography mask will be applied to all planes of the data array associated with the missing dimension(s). Suppose, for example, that the data being retrieved is a function of longitude, latitude and month, but the geography field is not a function of time. Then the same geography field will be applied to each month of data.

For land-ocean-seaice type geography data, EzGet assumes either the integers, 0, 1 , and 2 , or the floating point (real) numbers, $0.0,1.0$, and 2.0 , will be stored for land, ocean, and sea-ice grid cells, respectively. For the more detailed geography data, the table in Appendix B shows the integers that EzGet assumes have been stored in the geography file to define the various geographical regions.

\subsection{Subroutine defmisc}

This subroutine ('define miscellaneous') can be used to override certain default parameters assumed by EzGet (e.g., the value used to identify missing data, a parameter controlling the reporting of EzGet error messages, etc.). A call to defmisc is of the form:

call defmisc(param-name, var-type, param-value)

where

param-name (character string) indicates which parameter to define. The options are:

'input missing value' - to specify the value you expect missing data to be identified by on files you are reading. (If this parameter is not set, the default value is $1.0 \mathrm{e} 20$.)

'output missing value' - to specify the value you want missing data to be assigned after it is retrieved. (If this parameter is not set. the default value is $1.0 \mathrm{e} 20$. .)

'Iongitude name' - to assign an alias for the name EzGet will interpret as the longitude dimension. In this case param-value should be a character string. If this parameter is not set, the default value is 'longitude' (which already has aliases such as 'Ion', 'Longitude', and 'LONGITUDE' because EzGet 
is generally case insensitive and often only checks the first three characters of a string for equivalence). For example, if data have been stored with the longitude dimension named ' $x$ ', then defmisc should be called to inform EzGet that 'Iongitude' is also known as ' $x$ '. EzGet needs to know which dimension (if any) is longitude in order to correctly trap errors. (If this parameter is not set, the default value is 'Iongitude').

'latitude name' - to assign an alias for the name $\mathrm{EzG}$ Get will interpret as the latitude dimension. In this case param-value should be a character string. If this parameter is not set, the default value is 'Iatitude' (which already has aliases such as 'LATITUDE', 'Latitude', and 'lat' because EzGet is generally case insensitive and often only checks the first three characters of a string for equivalence). For example, if data have been stored with the latitude dimension named ' $y$ ', then defmisc should be called to inform EzGet that 'latitude' is also known as ' $y$ '. EzGet needs to know which dimension (if any) is latitude in order to correctly trap errors. (If this parameter is not set, the default value is 'latitude').

'data size' - to provide EzGet with the declared size of the data arrays passed either to subroutine getvdata or getfield. In this case paramvalue should be an integer. It is recommended that this parameter be set before calling subroutines getfield or getvdata. (If this parameter is not set, EzGet will not error exit if the data actually retrieved by these subroutines exceeds the declared array size. See Sections 3.16 and 3.18 for further information.)

'maximum dimension kept' - to limit the size of dimensions that will be stored by EzGet in a table for later retrieval. In this case param-value should be an integer. The size limit applies to the dimension length extracted by EzGet. not the dimension as it appears in the original file. (Compare with the 'Iongest dimension' option below.) Any dimension longer than this value must be specified as having 'unit' weighting. Furthermore, if the length exceeds the limit, you will be unable to obtain the values of the coordinates, weights or grid-cell edges by calling getcoord. getedges, or getdimwt. All longitude and latitude dimensions used in mapping data to a new grid or associated with geography files must be shorter than the limit. (If this parameter is not set, the default value is 2000 . If this limit is made too large (greater than about 2.5000) there is a risk that an absolute EzGet dimension limit will be exceeded.)

'Iongest dimension' - to reduce the storage required when accessing unusually long dimensions (usually associated with very long time-series). In this case param-value should be an integer. If a dimension is longer than the limit specified by 'longest dimension', then you must specify 'unit' weights for this dimension. The size limit applies to the number of elements stored in the original file, not the number of elements extracted for this 
dimension (compare with the 'maximum dimension kept' option above). If the length exceeds the limit. you will be unable to obtain the values of the coordinates, weights or grid-cell edges by calling getcoord. getedges, or getdimwt. All longitude and latitude dimensions used in mapping data to a new grid or associated with geography files must be shorter than the limit. (If this parameter is not set, the default value is 20000 . If this limit is made too large (greater than about 25000 ) there is a risk that an absolute EzGet dimension limit will be exceeded.)

'truncation' - to indicate to EzGet how many latitude zones span the globe from pole to pole for a spectral model. EzGet may need to know this in order to create correct gaussian weights when data being retrieved have been stored on a non-global domain. In this case param-value should be an integer. If EzGet retrieves data from a non-global domain, it is not necessary to set this parameter as long as the source data were stored on a global domain. (If this parameter is not set, EzGet assumes that the original latitude dimension spans the globe and creates gaussian weights accordingly.)

'error control' - to indicate how many errors detected by EzGet will be allowed before halting program execution and also whether or not error messages will be displayed. If param-value (an integer in this case) is less than or equal to 0 , then no messages will be displayed. If param-value equals 0 , the program execution will not be halted by EzGet no matter how many errors are encountered. If param-value is greater than 0 , then warnings and error messages will be displayed and execution will be halted when the the number of errors encountered equals the integer specified as param-value. (If this parameter is not set, EzGet will print errors and warnings and will halt execution after encountering 2 errors.)

'version' - to request that EzGet print (to your terminal) the date of release for the version of EzGet you are executing.

var-type (character string) indicates what type of variable ('real', 'integer'. or

'string') will be passed as the third argument (param-value). If for example, param-value is a character string (as it will be if param-name is 'longitude name' or 'latitude name'), then var-type should be set to 'string'.

param-value (real, integer, or character string) passes the value that will be assigned to the parameter selected through the first argument (param-name).

\subsection{Subroutine defregrd}

This subroutine controls mapping of data to another grid. The target grid can be 
defined by reference to another defined variable, or you can define it as a regular or gaussian grid by passing the appropriate subroutine arguments. Currently EzGet maps data to the target grid using an area-weighting alogrithm, which preserves area averages of the field. For correct mapping, you must define the spatial dimensions (with calls to defdim), correctly specifying the weight-type (for longitude, typically 'width' and for latitude, typically 'cosine' or 'gaussian', or in either case simply by specifying the acronym for one of the models appearing in Appendix A).

When this subroutine has been called, the data will be mapped to the target grid before returning it to you via getdata or getvdata. If the target grid is specified by an index to a defined variable, then the domain retrieved is determined by the domain specified for that defined variable (and the domain of the source grid is ignored). ${ }^{4}$ The type of weighting appropriate to the source grid and the specification of which dimension of the retrieved array varies most rapidly are always determined by the calls to defdim for the source data set. If you specify the target grid through the arguments of subroutine defregrd, then the domain is either obtained indirectly from those arguments or from the domain specified for the source grid, as discussed further below.

A call to defregrd is of the form:

call defregrd(var-index, target-cntrl, target-index, method, nlat, alat, dellat, nlon, alon, delion)

where

var-index (integer in the range 1 to 10 ) specifies which variable (defined previously or subsequently by a call to defvar), will be mapped to the new grid.

target-cntrl (character string) indicates how the target grid will be defined. The options are:

'none' - to turn off all mapping specifications so that the variable will remain on the source (i.e., original) grid.

'to' - to map data to the grid of the variable identified by target-index. The domain is determined by the calls to defdim for the variable referenced by target-index, so that you will receive the data as if it were originally stored on the target grid.

'uniform' - to map data to a user-defined grid that is evenly spaced in latitude and evenly spaced in longitude. The grid is defined by the last six arguments in the subroutine call (described below).

'gaussian' - to map data to a user-defined gaussian grid. The grid is defined by the last six arguments passed to this subroutine (as described below).

\footnotetext{
${ }^{4}$ Note that the domain will exactly concide with the range for the target grid, as if the source data had actually been stored on the target grid.
} 
target-index (integer in the range 1 to 10 ) specifies which variable defines the target grid. The latitude and longitude coordinates as well as the latitude and longitude domain will be taken from the variable referenced by target-index, as if the source data were actually stored on the target grid. If target-index $=0$, then no mapping will be done. If target-cntrl has not been set to 'to', then this argument is ignored. Be careful not to set target-index to a defined variable that might itself be mapped to a different grid, or you may encounter an error.

method (character string) specifies the interpolation method that should be used to map data to the new grid. Currently the only available method is an areaweighting algorithm, so method should be set to 'area-weighted'.

nlat (integer) controls the latitude domain of the target grid. To generate a gaussian grid, set nlat to the number of latitude grid cells spanning the globe from pole to pole (and set target-cntrl to 'gaussian'). In this case the actual domain will be determined by a call to defdim for the variable identified as var-index. To generate a uniformly spaced grid you may either: 1) set nlat to 0 , in which case the domain will be deterermined by a call to defdim for the variable identified as var-index, or 2) set nlat to the number of latitude grid cells you want to generate. In this second case, the first grid cell will be located at alat and the last grid cell will be located at alat+(nlat-1)*dlat (and the domain will extend half a grid cell beyond these locations). Thus for a uniformly spaced grid, if nlat>0, any latitude domain specifications made by calls to defdim are overridden. If target-cntrl has been set to 'to', then the value assigned to nlat is ignored.

alat (real) specifies the location of one of the latitude grid cells, but is completely ignored for gaussian grids. If nlat $>0$ and dlat $>0.0$, then alat will be the southernmost grid-cell generated; if $n l a t>0$ and dlat $<0.0$, then alat will be the northernmost cell. If nlat $=0$ then one of the target grid cells generated will be located at alat, but the domain of the data and the order that it will be retrieved are specified by calls to defdim (so, for example, even if dlat $>0.0$, data will be retrieved from north to south if in your call to defdim, bdry2 is specified to be less than bdry1). If target-cntrl has been set to 'to' or if a gaussian grid is specified. then this argument is ignored.

dlat (real) is the distance between neighboring latitude grid cells. but is completely ignored for guassian grids. This argument is also ignored if target-cntrl has been set to 'to'.

nlon (integer) controls the longitude domain of the target grid. You may either: 1) set $n$ lon to 0 . in which case the domain will be deterermined br a call to defdim for the variable identified as var-index. or 2) set nlon to the number of longitude grid cells you want to generate. In this second case. the first grid cell will be located at alon and the last grid cell will be located at alon+(nlon-1)*dlon (and the domain will extend half a grid cell beyond these locations). Thus if $n$ lon $>0$. 
any longitude domain specifications made by calls to defdim are overridden. If target-cntrl has been set to 'to', then the value assigned to nlon is ignored.

alon (real) specifies the location of one of the target longitude grid cells. If nlon>0 and $d l o n>0.0$, then alon will be the western-most grid-cell generated; if nlon>0 and $d l o n<0.0$, then alon will be the eastern-most cell. If $n l o n=0$ then one of the target grid cells generated will be centered at alon, but the domain of the data and the order that it will be retrieved are specified by calls to defdim (so, for example, even if dlon>0.0, data will be retrieved from east to west if in your call to defdim, bdry 2 is specified to be less than bdry1). If target-cntrl has been set to 'to', then this argument is ignored.

dlon (real) is the distance between neighboring longitude grid cells. If target-cntrI has been set to 'to', then this argument is ignored.

Although there are several ways to instruct EzGet to map data to a new grid as documented above, the four most common procedures are summarized here by way of example.

To map variable 1 (originally stored on a gaussian grid) to the grid of variable 2 (which is uniformly spaced in latitude and longitude):

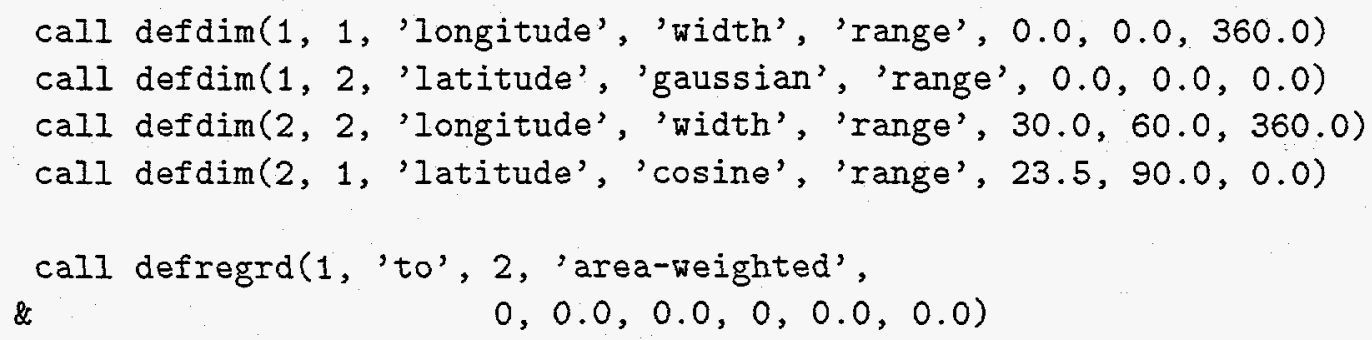

The domain is specified through the defdim calls for variable 2, which indicate that data should be retreived for the region bounded by $30^{\circ}$ and $60^{\circ} \mathrm{E}$ longitude and $23.5^{\circ}$ and $90^{\circ} \mathrm{N}$ latitude. Note that the order of the dimensions is determined by the defdim specifications for variable 1 , so that the data will be ordered with the longitude index varying most rapidly.

To map variable 1 (originally stored on a regular grid) to a different, uniformly spaced $10^{\circ}$ by $20^{\circ}$ latitude-longitude grid. but for a domain limited to the Northern Hemisphere and with latitudes stored from north to south:

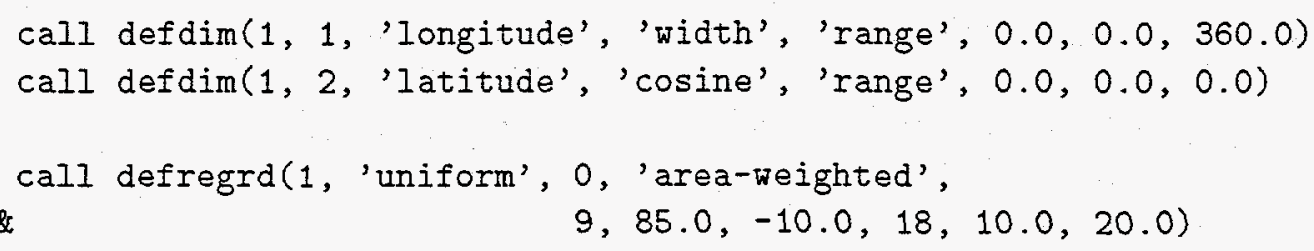


With this method of specifying the target grid, the domain boundaries coincide with grid cell edges (which lie half-way between the grid cell centers). In the above example, the domain extends from $90^{\circ}$ to $0^{\circ} \mathrm{N}$ latitude and $0^{\circ}$ to $360^{\circ} \mathrm{E}$ longitude. (The first latitude grid cell, for example, is $10^{\circ}$ wide and its center is at $85^{\circ} \mathrm{N}$, which means it extends from $90^{\circ} \mathrm{N}$ to $80^{\circ} \mathrm{N}$.)

To map variable 1 to a regular (uniformly spaced) $10^{\circ}$ by $20^{\circ}$ latitude-longitude grid, but for a domain specified through calls to subroutine defdim (which will provide more precise domain control than in the previous example):

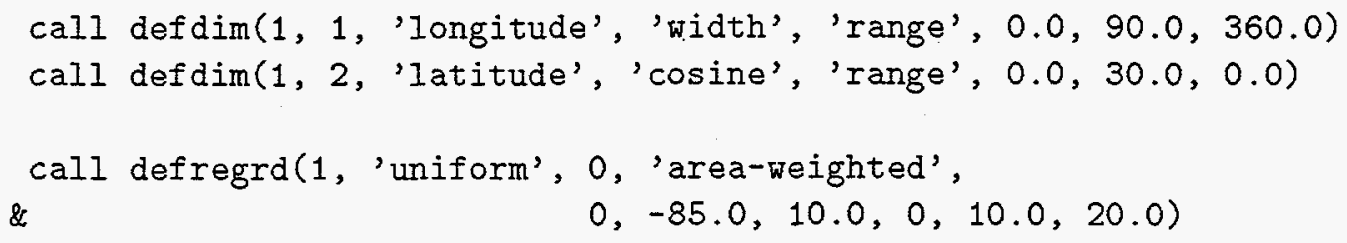

Note that unlike the previous example, the domain boundaries do not necessarily coincide with the edges of grid cells. In fact the last latitude grid cell (centered at $30 \mathrm{~N}$ ) extends from $25 \mathrm{~N}$ to $35 \mathrm{~N}$, but because the domain stops at $30 \mathrm{~N}$, the weights assigned this grid cell will be proportional to the area of a $5^{\circ}$ by $20^{\circ}$ cell, whereas the other grid cells will be assigned weights proportional to the area of $10^{\circ}$ by $20^{\circ}$ cells.

To map variable 1 (originally stored on a uniformly spaced grid) to a gaussian grid at $\mathrm{T} 42$ resolution:

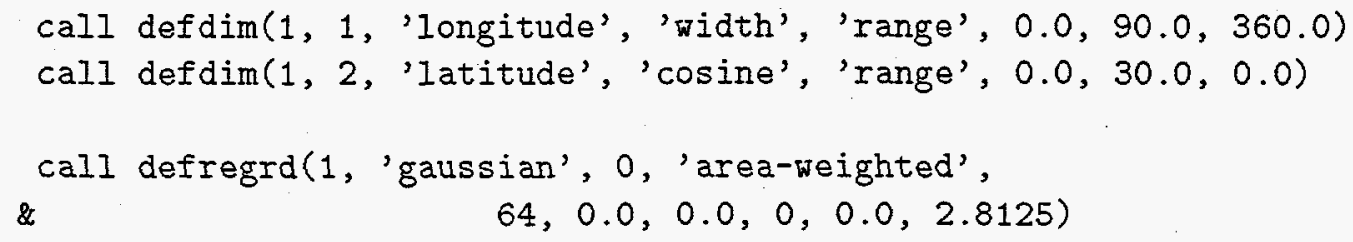

For gaussian grids the latitude domain is always set by a call to defdim. but the longitude domain is set by a call to defdim only if the number of longitudes specified in the call to defregrd is 0 , as it is in the above example.

\subsection{Subroutine defvar}

This subroutine defines a variable that can be referenced subsequently by the integer index you pass to EzGet as the first argument. A call to defvar is of the form:

call defvar(variable-index, variable-name, file-name)

where 
variable-index (integer in the range 1 to 10 ) is an index that will be used subsequently to identify the data defined by variable-name and file-name.

variable-name (character string of no more than 16 characters) is the name of the variable as it appears in the file from which data will be retrieved.

file-name ( character string of no more than 120 characters) is the full path (including file name) of the file that will be accessed by EzGet. (For files in DRS format, the '.dic' and '.dat' suffixes should be omitted, but for GrADS (or GRIB) files, the '.ctl' suffix should be included.)

If the name of the variable is not unique (i.e., more than one variable with the same name resides in the file being accessed by EzGet), then you must define the title and/or the source by calling subroutine defvarex as described below.

\subsection{Subroutine defvarex}

This subroutine is similar to defvar but must be used if more than one variable with the same name is stored in a file. In this case the "title" and/or the "source" of the data must be specified, so that EzGet can determine which field to retrieve. A call to defvarex is of the form:

call defvar(var-index, var-name, var-title, var-source, file-name)

where

var-index (integer in the range 1 to 10 ) that will be used subsequently to identify the data defined by var-name, var-title, var-source. and file-name.

var-name (character string of no more than 16 characters) is the name of the variable as it appears in the file from which data will be retrieved.

var-title (character string of no more than 80 characters) is the title of the variable as it appears in the file from which data will be retrieved. If you specify' ', then the title will be ignored in determining which variable to retrieve.

var-source (character string of no more than 120 characters) is the source of the variable as it appears in the file from which data will be retrieved. If you specify ' ' then the source will be ignored in determining which variable to retrieve.

file-name ( character string of no more than 120 characters) is the full path (including file name) of the file that will be accessed by Ez Giet. (For files in DRS format. the '.dic' and 'dat' suffixes should be omitted, but for Cr.ADS (or (iRIB) files, the '.ctl' suffix should be included.) 


\subsection{Subroutine domain}

This subroutine retrieves the number of dimensions, the names of the dimensions and the domain limits of each dimension as they appear in the file you are accessing with EzGet. The domain returned extends from the first coordinate value to the last coordinate value store in the file. This subroutine may be called after defining a variable (by defvar or devarex). A call to domain is of the form:

call domain(var-index, ndim, dimnames, beg, end)

where

var-index (integer in the range 1 to 10 ) specifies from which variable (defined by a calling defvar or defvarex) the domain information should be retrieved.

ndim (integer) returns the number of dimensions.

dimnames (character string, of 16 characters) returns a vector of length ndim that contains the names of the dimensions.

beg (real) returns a vector of length ndim that will contain the first coordinate value stored for each dimension.

end (real) returns a vector of length ndim that will contain the last coordinate value stored for each dimension.

\subsection{Subroutine domlimit}

This subroutine retrieves the domain limits of a single dimension and may be called after retrieving the data or calling subroutine shape. The domain limits as defined here extend from the leading edge of the first grid cell retritved to the trailing edge of the last grid cell retrieved. A call to domlimit is of the form:

call domlimit(var-index, dimname, beg, end)

where

var-index (integer in the range 1 to 10) specifies from which variable (defined by calling defvar or defvarex) the domain information should be retrieved.

dimname (character string. limited to no more than 16 characters) specifies the name of the dimension for which the domain limits are being requested.

beg (real) returns the leading edge of the domain for dimension dimname.

end (real) returns the trailing edge of the domain for dimension dimname. 


\subsection{Subroutine getcoord}

After data have been retrieved (or after shape has been called), this subroutine returns a vector of coordinate values of a specified dimension. The coordinate information refers to what has been or will be retrieved by EzGet, which may, for example, be a subset of what appears in the original file. A call to getcoord is of the form:

call getcoord(var-index, idim, coords)

where

var-index (integer in the range 1 to 10 ) specifies from which variable (defined by calling defvar or defvarex) coordinates should be retrieved.

idim (integer) is the dimension for which coordinates are being requested ( 1 for the first dimension of the variable, 2 for the second, etc.)

coords (real) is a vector that will receive the coordinates.

\subsection{Subroutine getdata}

This subroutine retrieves data, possibly mapping it to a different grid and masking user-specified geographical regions, and creates an appropriate mask (or set of "weights") associated with the data. It differs from getvdata in that the data are put into a multidimensional array structure and do not necessarily occupy contiguous memory. This subroutine checks whether the dimensions expected are correct or at least that the arrays are dimensioned large enough to accomodate the retrieved data.

A call to getdata is of the form:

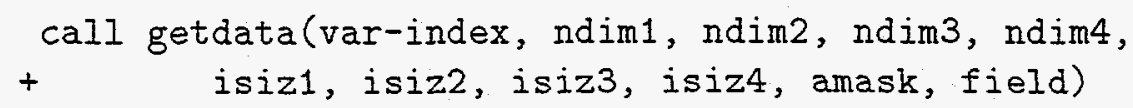

where, on entry to getdata.

var-index (integer in the range 1 to 10 ) specifies for which variable (defined by calling defvar or defvarex) data should be retrieved.

ndim1, ... ndim4 (integers) should be identical to the dimensions of amask and field. If these arrays have fewer than 4 dimensions. the unused dimensions should be set to either 0 or 1 . If set to 0 , then an error message will be displayed if the field actually has more dimensions than allowed for. For example. if field is dimensioned field $(64,32)$. then you should specify ndim $1=64$, ndim2 $=32$. ndim $3=0$. and ndim4=0. (Each of these arguments is left unaltered by 
EzGet, so for these first 4 arguements it is permissible to pass scalars defined in a. FORTRAN parameter statement or explicit scalar values.)

isiz1, ... isiz4 are what you expect the length of each dimension of the retrieved field to actually be. A warning will be provided if the dimensions of the retrieved field differ from what you expect. The warning for any dimension can be suppressed by assigning 0 to the corresponding isiz. (Each of these arguments might be reset by EzGet, so you must assiduously avoid passing scalars defined in a FORTRAN parameter statement or explicit scalar values. Instead of passing the explicit scalar ' $32^{\prime}$ ', you should assign the value ' 32 ' to some named integer' scalar and then pass the scalar.)

amask is a real array that will be filled (or partially filled) by "weights" associated with field. It can have $1,2,3$, or 4 dimensions, and these dimensions should be consistent with ndim1, ndim2, ndim3, and ndim4.

field is a real or integer array that will be filled (or partially filled) by the data identified by var-index. It can have $1,2,3$, or 4 dimensions, and these dimensions should be consistent with ndim1, ndim2, ndim3, and ndim4.

and, on return from getdata,

var-index will have been left unmodified.

ndim1, ... ndim4 will have been left unmodified.

isiz1, ... isiz4 will be the lengths of each dimension of the retrieved field $a s$ returned by getdata and thus will define the subdomain of the arrays that actually contain data.

amask will contain the "weights" associated with field. All elements outside the domain of the field retrieved by getdata will be set to 0 . If an element in the field array is "missing" or has been masked out, then the corresponding element in amask will also be 0 .

field will contain the data identified by var-index. All elements outside the domain of the field retrieved by getdata (i.e., if ndimi > isizi) will be set to 0 . "Missing data" within the domain retrieved will be set to the value defined by a call to defmisc or by default will be set to 1.e20.

\subsection{Subroutine getdimwt}

After data have been retrieved (or after shape has been called). this subroutine returns a vector containing the weights associated with a specified dimension. A call to getdimwt is of the form: 
call getdimwt (var-index, idim, wts)

where

var-index (integer in the range 1 to 10 ) specifies for which variable (defined by calling defvar or defvarex) weights should be retrieved.

idim (integer) is the dimension for which the weights are being requested ( 1 for the first dimension of the variable, 2 for the second, etc.)

wts (real) is a vector that will receive the weights associated with dimension idim.

\subsection{Subroutine getedges}

After data have been retrieved (or after shape has been called), this subroutine returns a vector containing the location of the grid-cell edges (i.e. boundaries) for a specified dimension. The coordinate information is consistent with what has been or will be retrieved by EzGet, which may, for example. be a subset of what appears in the original file. A call to getedges is of the form:

call getedges (var-index, idim, edges)

where

var-index (integer in the range 1 to 10 ) specifies for which variable (defined by calling defvar or defvarex) grid-cell information should be retrieved.

idim (integer) is the dimension for which grid-cell edges are being requested (1 for the first dimension of the variable, 2 for the second, etc.)

edges (real) is a vector that will receive the location of the grid-cell edges for dimension idim. This vector should be dimensioned at least 1 larger than the number of grid-cells retrieved.

\subsection{Subroutine getfield}

This subroutine simply retrieves data. but unlike getdata and getvdata it does no masking or mapping to a different grid. No mask is created and the data returned are forced to occupy contiguous memory. Before calling this subroutine it is recommended that you first call defmisc with the 'data size' option selected. informing EzGet of the actual cleclared size of the array that will receive the clata. This allows EzGet to check that your array is large enough to receive the data.

A call to getfield is of the form: 
call getfield(var-index, field)

where,

var-index (integer in the range 1 to 10 ) specifies from which variable (defined by calling defvar or defvarex) data should be retrieved.

field returns the data identified by var-index. "Missing data" within the domain retrieved will be set to the value defined by a call to defmisc or by default will be set to 1.e20.

\subsection{Subroutine getgeog}

This subroutine creates a geography mask for a specified region. Normally you do not need to create this mask as a separate step because defgeog will already have made it possible to select the desired geographical regions. The input geography data set that will be accessed may either be a simple land-ocean-seaice mask (or even more simply, a land-sea mask) or a more detailed geographical data set uniquely defining regions typically the size of a continent (as described in more detail in Section 3.5). Appendix B contains a list of regions identified on the standard geographical maps for AMIP and PMIP models available from PCMDI. A call to getgeog is of the form:

call getgeog(var-index, ndim1, ndim2, ndim3, ndim4,
$+\quad$ isiz1, isiz2, isiz3, isiz4, amask, select)

where

var-index (integer in the range 1 to 10 ) specifies which variable (defined by calling defvar or defvarex) contains the geography data.

ndim1, ... ndim4 (integers) should be identical to the dimensions of amask. If this array has fewer than 4 dimensions, the unused dimensions should be set to either 0 or 1 . If set to 0 , then an error message will be displaved if the field actually has more dimensions than allowed for. For example, if amask is dimensioned $\operatorname{amask}(64,32)$, then you should specify ndim1=64. ndim2=32, ndim3=0, and ndim $4=0$. (Each of these arguments is left unaltered by EzGet, so for these first 4 arguements it is permissible to pass scalars defined in a FORTRAN parameter statement or explicit scalar values.)

isiz1, . . isiz4 are what you expect the length of each dimension of the retrieved field to actually be. A warning will be provided if the dimensions of the retrieved field differ from what you expect. The warning for any dimension can be suppressed by assigning 0 to the corresponding isiz. (Each of these arguments might be reset by EzGet, so you must assiduously avoid passing scalars defined 
in a FORTRAN parameter statement or explicit scalar values. Instead of passing the explicit scalar ' 32 ', you should assign the value ' 32 ' to some named integer scalar and then pass the scalar.)

amask is a real array that on return indicates whether or not a grid cell lies within the selected region ( $\mathrm{a}^{\circ} 0.0^{\prime}$ indicates the cell lies outside the selected region and a '1.0' indicates it lies inside the region). amask can have $1,2,3$, or 4 dimensions, and the declared dimension lengths should be the same as ndim1, ndim2, ndim3, and ndim4.

select (character string) is a list of regions, separated by commas, specifying which geographical regions of the globe should be selected. If the geography data simply indicates regions of land, ocean, and possibly sea-ice (as stored in 'sft' files) then this argument might be, for example, 'ocean, seaice', which would select all regions except land. If the geography data contains the more detailed geographical information (as stored in sftbyrgn files), then this argument might be, for example, 'North America, South America, Greenland', which selects the major land areas of the western hemisphere. Note that the commas separating the listed regions may optionally be followed by blanks. For example, either 'Africa, Australia, Antarctica' or 'Africa, Australia, Antarctica' is acceptable. See Section 3.5 for further explanation.

\subsection{Subroutine getvdata}

This subroutine retrieves data. possibly mapping it to a different grid and masking user-specified geographical regions, and creates an appropriate mask (or set of "weights") associated with the data. It differs from getdata in that the data returned are forced to occupy contiguous memory. Before calling this subroutine it is recommended that you first call defmisc with the 'data size' option selected. informing EzGet of the actual declared size of the arrays that will receive the data and weights. This allows EzGet to check that your arrays are large enough to receive the data.

A call to getvdata is of the form:

call getvdata(var-index, amask, field)

where,

var-index (integer in the range 1 to 10 ) specifies from which variable (defined by calling defvar or defvarex) data should be retrieved.

amask returns the "weights" associated with field. If an element in the field array is "missing" or has been masked out. then the corresponding element in amask will also be 0 . 
field returns the data identified by var-index. "Missing data" within the clomain retrieved will be set to the value defined by a call to defmisc or by default will be set to $1 . e 20$.

\subsection{Subroutine initget}

This subroutine must be called to initialize $\mathrm{EzC}$ (iet. It assigns default values to a few parameters and sets up some internal tables. Subroutine initget has no arguments, so it is called as follows:

call initget

\subsection{Subroutine lendims}

After a field has been retrieved, this subroutine can return the length of each dimension of the retrieved variable. (The lengths returned give the dimensions of the data retrieved, which may not be the same as the data in the source file.) Normally this subroutine would be called after the field has been extracted by either subroutine getvdata or getfield. A call to lendims is of the form:

call lendims (var-index, $\operatorname{ldim} 1, \operatorname{ldim} 2, \operatorname{ldim} 3, \operatorname{ldim} 4$, isize)

where

var-index (integer in the range 1 to 10 ) specifies for which variable (defined by calling defvar or defvarex) dimension information should be retrieved.

$\operatorname{ldim} 1, \operatorname{ldim} 2, \operatorname{ldim} 3, \operatorname{ldim} 4$ (integers) returns the lengths of dimensions $1,2.3$. and 4 , respectively. If the second. third or fourth dimensions do not exist. then Idim for that dimension is set to 0 .

isize (integer) returns the size of the array retrieved $\left(=1 \operatorname{dim} 1^{*} \operatorname{ldim} 2^{*} \operatorname{ldim} 3^{*} \operatorname{ldim} 4\right.$. but with any 0 's replaced by 1 's).

\subsection{Subroutine shape}

Before EzGet retrieves data, this subroutine can obtain the length of each dimension of a variable. (The lengths returned indicate the size that will be retrieved. not 
necessarily the size stored.) Typically, this subroutine would be called in order to determine the size of the arrays that will be needed to accomodate the data that will be retrieved. A call to shape is of the form:

call shape(var-index, Idim1, Idim2, Idim3, Idim4, isize)

where

var-index (integer in the range 1 to 10 ) specifies for which variable (defined by calling defvar or defvarex) dimension information should be retrieved.

Idim1, Idim2, Idim3, Idim4 (integers) return the lengths of dimensions 1. 2, 3, and 4 , respectively. If the second, third or fourth dimensions do not exist, then Idim for that dimension is set to 0 .

isize (integer) returns the size of the array needed to accomodate the retrieved data (= $=\operatorname{dim} 1^{*} \operatorname{ldim} 2^{*} 1 \operatorname{dim} 3^{*} \operatorname{ldim} 4$, but with any 0 's replaced by 1 's).

\subsection{Subroutine varinfo}

This subroutine returns descriptive information retrievable from the file containing a defined variable. Information retrievable includes the data source, title, units, date, time, and variable-type. A call to varinfo is of the form:

call varinfo(var-index, param, info)

where

var-index (integer in the range 1 to 10 ) specifies from which variable (defined by calling defvar or defvarex) the information should be obtained.

param (character string) indicates what information should be returned. The options are:

'units' - to obtain the units of the variable ( 40 characters).

'source' - to obtain the source description for the variable (120 characters).

'title' - to obtain the title of the variable ( 80 characters).

'date' - to obtain the date the data were generated ( 8 characters).

'time' - to obtain the time the data were generated ( 8 characters).

'type' - type of variable (e.g., $\left.R^{*} 4, I^{*} 4, R^{*} 8, I^{*} 8, C^{*} 16\right)$ (8 characters).

'weight' - type of weighting (e.g., 'cosine', 'gaussian', 'uniform, etc.) (vector of 4 elements, each 8 characters long).

info (character string) returns the requested information. The length of the character string depends on what is specified for param. as indicated above. 


\section{$4 \quad$ Avoiding Errors}

\subsection{Input/output devices}

Because EzGet (through cdunif) opens files, it may internally assign and reference the following FORTRAN input/output device numbers: 91-94 and 96-99. You should therefore avoid using these same device numbers in your code (unless you have completed your calls to EzGet subroutines and have been careful to close all files opened by EzGet by calling subroutine closeget).

You should also note that the DRS library, which may be accessed by EzGet, has a limit of 6 DRS files that can be simultaneously open. Because EzGet might open up to 4 different DRS files, you should be careful to avoid opening more than 2 more DRS files (outside EzGet), or else the limit of 6 files will be exceeded.

\subsection{Subroutine and common names}

When you write programs that will be linked to EzGet, make sure that the names identifying your subroutines, functions and commons are different from names already used in EzGet. Specifically avoid the following names:

- Commons: cdimtbl, cdomain, ciotbl. cregddim, cscalars, cvartbl, usersget

- Integer functions: applywts, doregrid, genwts, getdimen, getfld, mkdimtbl, opendrs, univunit, xgeog. xregion

- Logical functions: caseindp

- Subroutines: bsslzr, closeget, clrtable, defdim, defdimi, defgeog, defmisc, defregrd, defvar. defvarex. domain, domlimit, errcheck, gauaw, getcoord, getdata getedges, getdimwt, getfield. getgeog, getvdata. initget, lendims, maparea. rgdarea, shape, varinfo

- Combined List: applywts, bsslzr. caseindp. cdimtbl, colomain. cjotbl. closeget. clrtable, cregddim. cscalars, crartbl. defdim. defdimi, defgeog, defmisc defregrd. defvar. defvarex, domain. domlimit, doregrid. errcheck. gauaw, genwts. getcoord. getdata. getdimen. getdimwt, getedges, getfield. getfld. getgeog. getvdata. initget. lendims, maparea, mkdimtbl, opendrs. rgdarea, shape, univunit, usersget. varinfo. xgeog, xregion 


\subsection{EzGet size limits}

To limit the amount of memory required in running EzGet, there are limits on how many different variables and dimensions can be simultaneously accessed. The maximum number of variables that can be defined is 10 (which is why the index in a call to def var is limited to the range 1 to 10 ). The maximum number of different dimensions that will be accomodated by EzGet is 100 . This limit is usually sufficient unless you loop through some long dimension, each time extracting a single plane. For example if you have a 10-year long time-series of monthly data of surface air temperature and you cycle through the individual months, then when you get to month 99 , you will encounter an error because you already will have saved 100 dimensions (the latitude and longitude dimensions plus 98 time dimensions, one for each of the first 98 months). If, on the other hand, you had extracted the full three-dimensional array at one time, then you would have generated only 3 dimensions (2 spatial dimensions and 1 time dimension). You may make room for more dimensions by calling subroutine clrtable at some convenient point.

There are also limits on how many coordinate values can be extracted and placed in a table for future reference by EzGet. See subroutine defmisc for further information.

\section{$5 \quad$ Obtaining and Installing EzGet Software}

The EzGet software is currently available for the following platforms/operating systems: ${ }^{5}$

- Sun/SunOS 4.1.3

- Sun/Solaris 2.4

- IBM RS6000/AIX 3.2

- HP/HP-IX 9.0

- SCII Irix 5.3

You may obtain the FORTRAN libraries comprising EzGet from the PCMDI web site:

${ }^{5} \mathrm{EzG}$ (Get will also be ported to the (ray/Cnicos and DEC Alpha/OSF on request. 
home page: http://www-pcmdi.llnl.gov/

EzGet location: http://www-pcmdi.llnl.gov/ktaylor/ezget/ezget.html

In addition, if you will be reading netCDF files, you will need to acquire the netCDF library from the unidata web site:

$\mathrm{ftp}: / / \mathrm{ftp}$.unidata.ucar.edu/pub/netcdf/

There are geography data sets available for the AMIP and PMIP models that allow you to extract data from specific geographical regions (e.g., North America, South Atlantic, Australia, etc.). Information on how to obtain these geography data sets along with copies of the examples given in section 2 are available at the PCMDI web site.

\section{Acknowledgments}

I thank Peter Gleckler, Ben Santer, and Curt Covey for exercising early versions of this software and uncovering several bugs; I appreciate their patience and feedback. I also thank them and Jean-Yves Peterschmitt, Emannuelle Cohen-Solal, and Larry Gates for reading parts of this documentation and making comments and suggestions to improve it. Bob Drach (the author of cdunif) has been helpful in several ways, including providing assistance in porting this software to different platforms.

This work was performed under the auspices of the U.S. Department of Energy Environmental Sciences Division by the Lawrence Livermore National Laboratory under contract No. W-7405-ENG-48. 


\section{Appendix A}

TABLE 1. The AMIP and PMIP model acronyms given below are recognized by EzGet and may be used in calls to subroutines defdim or defdimi to specify "weights" for longitude and latitude. The weights may be needed to map data to a different grid or may be used to compute area-weighted statistics. The user may specify the correct weights either by the model acronym shown in column one below, or more explicitly by specifying the options indicated in the third and fourth columns below.

\begin{tabular}{|c|c|c|c|}
\hline $\begin{array}{c}\mathrm{E} z \mathrm{Giet} \\
\text { Acronym }^{1}\end{array}$ & AMIP/PMIP Group & $\begin{array}{l}\text { Longitude } \\
\text { Weights }\end{array}$ & $\begin{array}{l}\text { Latitude } \\
\text { Weights }\end{array}$ \\
\hline 'bmr*' & $\begin{array}{l}\text { Bureau of Meteorology Research Centre } \\
\text { (BMRC) }\end{array}$ & 'width' & 'gaussian' \\
\hline 'ccc*' & $\begin{array}{l}\text { Canadian Centre for Climate Modelling } \\
\text { and Analysis (CCCMA) }\end{array}$ & 'width' & 'gaussian' \\
\hline 'ccm*' & $\begin{array}{l}\text { various groups and versions of the Com- } \\
\text { munity Climate Model }\end{array}$ & 'width' & 'gaussian' \\
\hline 'cos*' & $\begin{array}{l}\text { Center for Climate System Research } \\
\text { (CCSR) }\end{array}$ & "width" & 'gaussian' \\
\hline 'cnr*' & $\begin{array}{l}\text { Centre National de Recherches } \\
\text { Météorologiques (CNRM) }\end{array}$ & 'width' & 'gaussian' \\
\hline$' \operatorname{col} *$ ' & $\begin{array}{l}\text { Center for Ocean-Land--ttmosphere Stud- } \\
\text { ies (COLA) }\end{array}$ & 'width' & 'gaussian' \\
\hline$' \operatorname{csi} i{ }^{\prime}$ & $\begin{array}{l}\text { Commonwealth Scientific and Industrial } \\
\text { Research Organization (CSIRO) }\end{array}$ & 'width' & 'gaussian' \\
\hline 'csu*' & Colorado State Eniversity (CSU) & 'width' & 'csu', \\
\hline 'der*' & $\begin{array}{l}\text { Dynamical Extended Range Forecasting } \\
\text { (at GFDL) }\end{array}$ & 'width' & 'gaussian' \\
\hline 'dnm*' & $\begin{array}{l}\text { Department of Numerical Mathematics } \\
\text { (of the Russian Academy of Sciences) }\end{array}$ & 'width' & 'cosine' \\
\hline 'ecm*' & $\begin{array}{l}\text { European Centre for Medium-Range } \\
\text { Weather Forecasts (ECMWF) }\end{array}$ & 'width' & 'gaussian' \\
\hline 'gen*' & $\begin{array}{l}\text { Various groups and versions of the GEN- } \\
\text { ESIS model }\end{array}$ & 'width' & 'gaussian' \\
\hline
\end{tabular}




\begin{tabular}{|c|c|c|c|}
\hline $\begin{array}{c}\text { EzGet } \\
\text { Acronym } 1 \\
\end{array}$ & AMIP/PMIP Group & $\begin{array}{l}\text { Longitude } \\
\text { Weights }\end{array}$ & $\begin{array}{l}\text { Latjtude } \\
\text { Weights }\end{array}$ \\
\hline 'gfd*' & $\begin{array}{l}\text { Geophysical Fluid Dynamics Laboratory } \\
\text { (GFDL) }\end{array}$ & 'width' & 'gaussian' \\
\hline 'gis*' & $\begin{array}{l}\text { Goddard Institute for Space Studies } \\
\text { (GISS) }\end{array}$ & 'width' & 'cosine' \\
\hline 'gla*' & $\begin{array}{l}\text { Goddard Laboratory for Atmospheres } \\
\text { (GLA) }\end{array}$ & 'width' & 'cosine' \\
\hline 'gsf*' & Goddard Space Flight Center (GSFC) & 'width' & 'cosine' \\
\hline 'iap*' & $\begin{array}{l}\text { Institute of Atmospheric Physics (of the } \\
\text { Chinese Academy of Sciences) }\end{array}$ & 'width' & 'cosine' \\
\hline 'jma*' & Japan Meteorological Agency (JMA) & 'width' & 'cosine $e^{3}$ \\
\hline 'Imc*' & $\begin{array}{l}\text { various version of the LMD model used by } \\
\text { the Laboratoire de Modélisation du Cli- } \\
\text { mat et de } 1^{\prime} \text { Environnement (LMCE) }\end{array}$ & 'width' & 'Imc'4 \\
\hline 'Imd*' & $\begin{array}{l}\text { Laboratoire de Météorologie Dynamique } \\
\text { (LMD) }\end{array}$ & 'width' & 'Imd'4 \\
\hline 'mgo*' & Main Geophysical Observatory (MGO) & 'width' & 'gaussian' \\
\hline 'mpi*' & $\begin{array}{l}\text { Max-Planck-Institut für Meteorologie } \\
\text { (MPI) }\end{array}$ & 'width' & 'gaussian' \\
\hline 'mri*' & Meteorological Research Institute (MRI) & 'width' & 'cosine' \\
\hline 'nca*' & $\begin{array}{l}\text { National Center for Atmospheric Research } \\
\text { (NCAR) }\end{array}$ & 'width' & 'gaussian' \\
\hline 'nme*' & National Meteorological Center (NMC) & 'width' & 'gaussian' \\
\hline 'nrl*' & Naral Research Laboratory (NRL) & 'width' & 'gaussian' \\
\hline 'rpn*' & Recherche en Prévision Numérique (RPN) & 'width' & 'gaussian' \\
\hline 'sng*' & $\begin{array}{l}\text { State University of New York at Albany / } \\
\text { National Center for Atmospheric Research } \\
\text { (SUNYA/NCAR) }\end{array}$ & 'width' & 'gaussian' \\
\hline 'sun*' & $\begin{array}{l}\text { State University of New York at Albany } \\
\text { (SLNYA) }\end{array}$ & 'width' & 'gaussian' \\
\hline 'ucl*' & $\begin{array}{l}\text { Eniversity of California at Los Angeles } \\
\text { (UCLA) }\end{array}$ & 'width' & 'ucl,'2 \\
\hline
\end{tabular}




\begin{tabular}{clcc}
$\begin{array}{c}\text { EzGet } \\
\text { Acronym }\end{array}$ & \multicolumn{1}{c}{ AMIP/PMIP Group } & $\begin{array}{c}\text { Longitude } \\
\text { Weights }\end{array}$ & $\begin{array}{c}\text { Latitude } \\
\text { Weights }\end{array}$ \\
\hline 'uga*' & $\begin{array}{l}\text { The UK Universities (ilobal Atmospheric } \\
\text { Modelling Programme (UGAMP) }\end{array}$ & 'width' & 'gaussian' \\
'uiu*, & $\begin{array}{l}\text { University of Illinois at Urbana-Cham- 'width' } \\
\text { paign (UIUC) }\end{array}$ & 'cosine' \\
'ukm*' & $\begin{array}{l}\text { United Kingdom Meteorological Office } \\
\text { (UKMO) }\end{array}$ & 'width' & 'cosine' \\
'yon*' & Yonsei University (YONU) & 'width' & 'cosine' \\
\hline
\end{tabular}

${ }^{1}$ The model acronyms are truncated by EzGet to three characters so in the table above the character '*' is 'wild' meaning that it represents any string of characters, including the null string.

'The UCLA and CSU models have regularly spaced latitudes, but there is no grid point at the poles, so the most poleward grid cells in each hemisphere have a latitudinal width of 1.5 times the other grid cells. For proper construction of area weights, specify 'csu' or ' $u c l$ ' (not 'cosine') for the latitude weights.

${ }^{3}$ The JMA model has a gaussian grid, but the data are reported on a $2.5 \times 2.5$ degree regular grid.

${ }^{4}$ The LMD and LMCE models have equal area grid cells which become elongated in latitude away from the equator. For proper construction of area weights, specify 'Imd' or 'Imc' for the latitude weights. 


\section{Appendix B}

TABLE 2. Specifications for Geographical Regions

\begin{tabular}{|c|c|c|}
\hline Integer I.D. & Region & EzGet Specification \\
\hline 0 & ocean & 'oce*' \\
\hline 1 & land & 'Ian*' \\
\hline 2 & sea ice & 'seai*', 'sea-i*', 'sea i*' \\
\hline 217 & North America & 'n* ame*' \\
\hline 216 & South America. & 's* ame*' \\
\hline 215 & Greenland & 'gre*' \\
\hline 218 & Africa & 'afr*' \\
\hline 219 & Europe \& Asia & 'eur*asia*' \\
\hline 221 & Australia & 'aus*' \\
\hline 220 & Antarctica & 'ant*' \\
\hline 222 & Indo Pacific Islands & 'ind* i*' \\
\hline 211 & American Lakes & 'ame* 1*' \\
\hline 212 & Baffin \& Hudson_Bays & 'baf*' \\
\hline 208 & Asian \& African Lakes & 'asi* 1*' \\
\hline 207 & Mediterranean Sea & 'med*' \\
\hline 201 & North Pacific Ocean & 'n* pac*' \\
\hline- & South Pacific Ocean & 's* pac*' \\
\hline 202 & South Pacific (N. of Melbourne) & 's* pac* 1*' \\
\hline 203 & $\begin{array}{c}\text { South Pacific (N. of Cape Horn } \\
\text { and S. of Melbourne) }\end{array}$ & 's* pac* 2*' \\
\hline 204 & South Pacific (S. of Cape Horn) & 's* pac* 3*' \\
\hline 209 & North Atlantic Ocean & 'n* atl*' \\
\hline- & South Atlantic Ocean & 's* atl*' \\
\hline 213 & $\begin{array}{c}\text { South Atlantic (N. of Cape of } \\
\text { Good Hope) }\end{array}$ & 's*atl* 1*' \\
\hline 214 & $\begin{array}{c}\text { South Atlantic (S. of Cape of. } \\
\text { Good Hope) }\end{array}$ & 's* atl* 2*' \\
\hline- & Indian Ocean & 'ind*' \\
\hline 205 & Indian Ocean (N. of S. Australia) & 'ind* $1 *$ ' \\
\hline 206 & Indian. Ocean (S. of S. Australia) & 'ind* $2 *$ ' \\
\hline 210 & Arctic Ocean & 'arc*' \\
\hline
\end{tabular}

Notes concerning Table 2 :

- The character ' $*$ ' is 'wild 'meaning that it represents any string of characters, including the null string (but blanks are not permitted except as part of the group, ' \& '). For example, EzGiet treats the following strings as equivalent: 'North America', 'n ame'. 
'N. Amer.', etc.

- EzGet is case insensitive (at least in interpreting the geography strings) so. for example, the following strings are equivalent: 'North America', 'north america', 'NORTH AMERICA', etc.

- It is permissible to follow the name of an ocean or sea by the strings ' ocean' or ' sea'. For example, 'Mediterranean Sea' is equivalent to 'Mediterranean' and 'North Pacific Ocean' is equivalent to 'North Pacific. Note, however, that the numbered ocean basin subdomains (e.g., South Pacific 1') should not be followed by either' ocean' or' sea'.

- The following equivalences are recognized:

'S Pac' = 'S Pac 1, S Pac 2, S Pac 3'

'S AtI' = 'S Atl 1, S Atl 2'

'Indian' = 'Indian 1, Indian 2'

- When accessing the detailed geography data, you cannot select 'seaice' because the sea-ice distribution is not available from those files. All land and ocean regions can, however, be selected because the following equivalences are recognized:

'Iand' = 'n ame, s ame, gre, afr, eur-asia, aus, ant, ind $i$ '

'ocean' = 'ame 1 , baf, asi 1 , med, $n$ pac, $s$ pac, $n$ atl, $s$ atl, ind, arc'

- The entry '-' appears in column one of the table because the region comprises two or more subregions. For example, 'South Atlantic' selects all grid cells in either the 'South Atlantic 1' or the 'South Atlantic 2' regions (i.e., all grid cells that have been assigned either the value 213 or the value 214 in the geography file).

Figure 1. which follows on the next page, shows the geographical regions recognized by EzGet. The Indo-Pacific Islands. American Lakes, and Asian \& African Lakes can also be selected as indicated in the table, but are not labeled on the figure. 


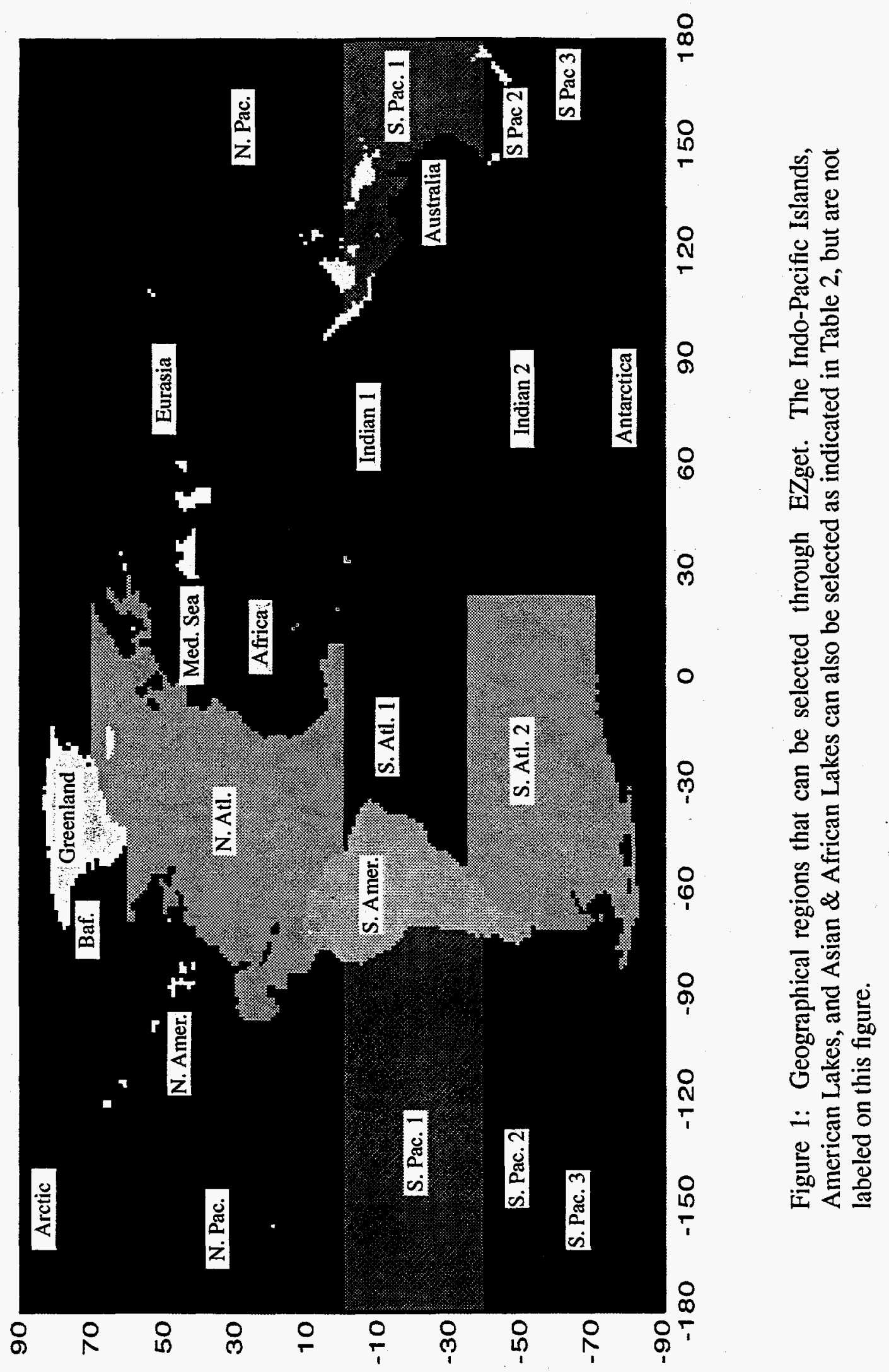




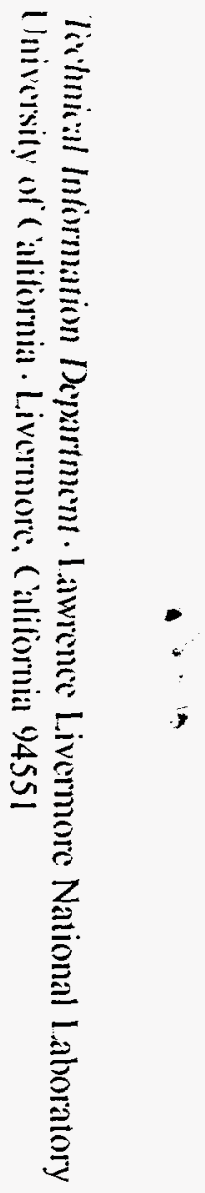

